\title{
Skopische Sozialität
}

\section{Sichtbarkeitsregime und visuelle Praktiken im Boxen ${ }^{1}$}

\author{
Von Christian Meyer und Ulrich von Wedelstaedt
}

Zusammenfassung: Der Text untersucht die Vielschichtigkeit und Vielgestaltigkeit visueller Praktiken in der sozialen Interaktion am Beispiel des Boxens. Beim Boxkampf scheint es sich zunächst um eine körperliche Auseinandersetzung zwischen zwei Beteiligten zu handeln, bei welcher der physischen Kraft und Geschicklichkeit maßgeblicher Einfluss auf Sieg oder Niederlage zukommt. In der vorliegenden Fallstudie wird jedoch aufgezeigt, dass auch visuelle Praktiken verschiedener am Kampfgeschehen beteiligter Personen großen Anteil am Erfolg haben. Diese Praktiken beziehen sich auf verschachtelte und sich überlagernde ,skopische Regime“ im Boxsport, die jeweils spezifische normative Erwartung an die Sichtbarmachung von Handlungen ebenso wie an deren Verdeckung stellen. Hierzu zählt sowohl die ostentative Umsetzung der permanent vokal in den Ring gerufenen Anweisungen des Trainers durch den Boxer als auch dessen demonstrative, an den Ringrichter adressierte Einhaltung der Boxregeln, seine betonte Performanz des Nichtgetroffenseins beim Schlag des Gegners in Richtung des Punktrichters und seine Zurschaustellung von sportlicher Fitness, moralischer Integrität und unerschrockener Vitalität gegenüber den Zuschauern. All diese Sichtbarkeiten müssen, jeweils adressatenspezifisch, vom Boxer in seinen Einzelhandlungen gewissermaßen ,aufgeschichtet“ werden. Nicht zuletzt handelt es sich beim Sehen im Bereich des Boxgeschehens selbst sogar um eine zwischen Athlet und Trainer verteilte Tätigkeit, die Sichtbarkeiten, aber auch körperliche Aktivitäten im Hinblick auf die visuellen Erwartungen aller anderen Anwesenden (Gegner, Richter, Zuschauer) manipuliert. Sehen wird so zu einem intersubjektiven, sozialen Gut, das kooperativ erzeugt wird.

\section{Zur Soziologie skopischer Praktiken - theoretische Vorbemerkungen}

Die visuelle Soziologie kann sich auf eine ganze Reihe unterschiedlicher Strömungen berufen, die das Visuelle hervorgehoben haben. Für diesen Aufsatz sind insbesondere die Visual Studies und die ethnomethodologische Erforschung visueller Praktiken von Bedeutung.

Die Visual Studies haben seit mehr als 20 Jahren konstant an Bedeutung gewonnen. Insbesondere die philosophische Kritik am sogenannten Okulozentrismus der Moderne - ihrer vermeintlichen visuellen Zentriertheit oder gar optizistischen Obsession (vgl. z.B. Tyler 1984) hat eine Reihe von neuen Forschungsthematiken inspiriert, darunter etwa ein wiedererstarktes Interesse an der Kunst als bildlicher Sphäre, an bildlastigen neuen Medien oder bildgebenden Verfahren. Die Hypothese der Visual Studies ist, dass Visualität, optische Wahrnehmung und Bildlichkeit nichts Naturgegebenes und kein direktes Abbild der Natur sind (vgl. Rorty 1981), sondern ein Resultat sozialer Konstruktionen darstellen. Was wir sehen, ist stets von vornherein kulturell geprägt und sozial gestaltet. Methodologisch ist für die Visual Studies ein Wille zur ikonoklastischen Kritik des Verführungscharakters von Bildern leitend (vgl. als Bestandaufnahme Smith 2008 sowie kritisch Mitchell 2002).

Neben den Visual Studies haben ethnomethodologisch und konversationsanalytisch orientierte Forscher eine Soziologie visueller Praxis entwickelt (vgl. Macbeth 1999; Goodwin 2000 b). Aus der Erfahrung beider Strömungen heraus können einige theoretische Grundorientierungen genannt werden, welche die im Folgenden vertretene Soziologie visueller Praxis anleiten.

1 Wir danken den Herausgebern dieses Sonderheftes sowie zwei anonymen Gutachtern für wertvolle Hinweise zu einer früheren Version dieses Textes. 
$\mathrm{Zu}$ diesen Orientierungen zählt, dass an Stelle sehr pauschaler sensorischer Zentriertheiten wie des viel beschworenen generellen Okulozentrismus der Moderne vielmehr die Differenziertheiten ,visueller Subkulturen“ (Jay 1988: 4) und situativer ,,professioneller Blickwinkel“ (Goodwin 1994) in das Zentrum des Forschungsinteresses gerückt werden. Sehen erfordert visuelle Gewohnheiten, denen stillschweigende kulturelle Regeln unterliegen, und situierte Kontexte, die optische Reize in Seherlebnisse transformieren. Aus diesem Grund erscheint es sinnvoll, sehr differenziert von unterschiedlichen visuellen Subkulturen mit unterschiedlichen, möglicherweise situativ variierenden optischen Gewohnheiten und Praktiken auszugehen. Das Ensemble solcher subkulturell dominanten Gewohnheiten wurde bereits als ,skopisches Regime" (Metz 1982) bezeichnet. Ein solches besteht aus vorherrschenden kulturellen Konventionen, die das, was wir sehen, mit dem verbinden, wie wir sehen (vgl. Goodwin 2000 b; Foucault 1976 und Goodwin / Goodwin 1997 für interessante Fallstudien). ${ }^{2}$ Skopische Regime steuern unsere Aufmerksamkeit in einer Weise, dass unsere Wahrnehmungen und deren Interpretation spezifische Inhalte aus dem „Rohmaterial“ der optischen Reize entstehen lassen. Insofern wird die „Zweite Natur“ des skopischen Regimes zu einem Wirklichkeitsfilter: es macht bestimmte Aspekte unsichtbar und lässt andere optisch hervortreten (vgl. Goodwin 2000 b: 165). Die Idee des skopischen Regimes ist damit zentral für ein Verständnis davon, wie soziales Handeln und kulturelle Orientierungen erschaffen werden, wenn der Sehsinn beteiligt ist.

Ethnomethodologische Forschungen haben demgegenüber weniger auf die soziale Konstruktion des Visuellen, sondern - komplementär dazu - auf die visuelle Konstruktion von Sozialität fokussiert (vgl. dazu Goodwin 2000 b; Mitchell 2002: 170 f). Nicht nur die Arbitrarität und Konstruiertheit eines scheinbar natürlich gegebenen Visuellen, sondern auch zugleich die Funktionen und Bedeutungen von Visualität für die Hervorbringung des Sozialen werden hier berücksichtigt. Denn gerade die scheinbare Natürlichkeit und Durchsichtigkeit visuell konstruierter Sozialität (vgl. Hester / Francis 2003; in Bezug auf Texte Wolff 1994) dient auch der Hervorbringung und Verstetigung skopischer Regime, welche interaktionale und kollaborative Praktiken anleiten, kulturelle Normalität und Rationalität hervorbringen und die Zugehörigkeit von Personen zu Gruppen oder Praxisgemeinschaften bestätigen und verfestigen.

An noch prominenterer Stelle zählt zu einem Fokus auf die visuelle Konstruktion von Sozialität die Konzentration auf die sozialen Praktiken, die mit dem Gebrauch und der Diskursivierung des Visuellen verbunden sind (vgl. Goodwin 2000 b: 166; Levin 1988: 14). Beides, Redeweisen wie Sehweisen, sind eng verwoben und werden wechselseitig von den Konventionen und Praktiken der sozialen Gemeinschaft gestaltet. Hierzu zählen z.B. Praktiken des Sehens und Zeigens, vor allem derjenigen, die uns unmittelbar und unvermittelt und damit in besonderer Weise evident erscheinen (vgl. dazu Kita 2003, darin insbesondere Goodwin 2003,

2 Dabei können nicht nur einzelne visuelle Praktiken und skopische Regime beschrieben, sondern auch nebeneinander existierende oder aufeinander folgende Praktiken, Regime und Subregime unterschieden und in ihrem Unterschied bewertet werden, wie etwa das des Theaters im Kontrast zum Kino (Metz 1982: 61ff) oder der „static, unblinking, and fixated“ Blick des cartesianischen Perspektivismus der italienischen Renaissance im Vergleich zur zeitgenössischen holländischen oder zur Barockkunst, deren Auge demgegenüber „dynamic, moving [...] from one focal point to another“ (Jay 1988: 7) ist. 
sowie das „showing seeing“ bei Mitchell 2002: 177ff). Es geht also weniger um die Bilder selbst, als um deren soziale Einbettung, ihre „lives and loves“ (Mitchell 2002: 170). ${ }^{3}$

Eine praxistheoretisch angeleitete visuelle Soziologie ist bei all dem allerdings darauf bedacht, keinen isolierten Blick auf den Sehsinn und dessen soziale Praktiken und kulturelle Produktivitäten zu werfen. Vielmehr versteht sie ihr Forschungsfeld multimodal und begreift visuelle in ihrem Zusammenspiel mit anderen sensorischen Praktiken, die jeweils spezifische semiotische Eigenschaften besitzen (vgl. Goodwin 2000 b). So sagt Jay über das skopische Regime des Barock: „,baroque visual experience has a strongly tactile or haptic quality, which prevents it from turning into the absolute ocularcentrism of its Cartesian perspectivalist rival " (Jay 1988: 17). Allgemeiner ausgedrückt hat eine praxistheoretisch angeleitete visuelle Soziologie immer auch ein Interesse an unsichtbaren, ungesehenen, blinden, übersehenen und unsehbaren Phänomenen als komplementären Gegenstücken zur optizistischen Ideologie. Als eine Soziologie der Sinne bedenkt sie das Taktile, Hörbare, Haptische sowie Geruch und Geschmack als ihr Anderes ebenso mit wie die Integrationsleistung verschiedener Modalitäten (vgl. Goodwin 2000 b: 166; Mitchell 2002: 170, 174). Die Ethnomethodologie hat mit ihrem Begriff der Accountability darauf aufmerksam gemacht, dass die Maßnahmen, mit denen soziale Akteure sich ihre Handlungen und Praktiken wechselseitig verstehbar machen, nicht nur visuelle, sondern grundsätzlich alle verfügbaren semiotischen Modalitäten umfassen können. Zudem gehen soziale Akteure mit skopischen und anderen Beobachtungspraktiken ihrer Gegenüber bzw. ihres Kontexts immer aktiv um. Soziales Tun umfasst daher immer auch eine aktive Orientierung an bestehenden skopischen Regimen und die Verstehbarmachung gegenüber deren Repräsentanten, was ihm einen grundlegend reflexiven Charakter verleiht. Für skopische Regime bedeutet das, dass den an ihnen orientierten skopischen Praktiken von beobachtenden Instanzen - gewissermaßen als deren Kehrseite - immer auch die Sichtbarkeitsregime und -praktiken der Beobachteten gegenüberstehen - ein Aspekt, den Foucault (1976) übersehen hat.

Ethnomethodologische und konversationsanalytische Forschungen haben daher stets hervorgehoben, dass visuelle Praxis erstens als gemeinsame Aktivität ausgeübt wird und damit interaktional-reflexiv ist (z.B. Goodwin 1996). Sehen ist kein individueller neuro-physiologischer Prozess, sondern es geht bei der visuellen Konstruktion von Sozialität häufig um adressiertes, an Wechselseitigkeit orientiertes Zeigen von Sehen, Blicken und Schauen. So hat Goodwin (1980) die fundamentale Rolle des Blickverhaltens der Teilnehmer für die Koordination von Interaktionen herausgearbeitet. Darüber hinaus zeigen sie sich über Blicke an, welche Ereignisse sie in einem spezifischen Moment für relevant erachten und worauf sie die Aufmerksamkeit des Gesprächspartners lenken möchten. Interaktion ist also nicht nur über

3 Bei der Betrachtung visueller Praktiken können auch Medien eine Rolle spielen, wie etwa bei Knorr Cetina (2012), die von einer Ausbreitung skopischer Medien ausgeht, die ferne audiovisuelle Phänomene situativ präsent werden lassen, entlegene Räume und Wissenswelten vermitteln sowie die Grenzen zwischen der Situation und ihrem Umfeld verschieben. Skopische Medien verändern die Formen sozialer Praxis und Interaktion hin zu einer, wie sie es nennt, ,synthetischen Interaktion“, indem sie Face-to-face-Beziehungen in Face-to-screen-Beziehungen und Formen der kopräsenten Koordination in skopische Koordinationsformen verwandeln. Insgesamt besteht die Annahme darin, dass mit den neuen (eben zumeist skopischen) Medien die Welt zunehmend visuell und informational statt wie zuvor materiell und natürlich gestaltet würde. Die intensive visuelle Interaktivität der neuen Medien und die wachsende Bedeutung von internet-basierten Arbeitsplattformen, Kollaborationswerkzeugen und Rollenspielen haben mithin die Entstehung einer veränderten skopischen Sozialität gefördert. Während in der Welt der materiell-körperlichen Kopräsenz die Sehende eine aktive Teilnehmerin an der soziovisuellen Situation ist, ist sie in der skopischen Welt nur noch passive Rezipientin (oder im besten Falle, Rekonstrukteurin) von dekontextualisierten Bildbedeutungen. Diese Diagnose stützt nicht selten kulturpessimistisch ausgerichtete Befürchtungen populärwissenschaftlicher Medienkritiker (z.B. Spitzer 2006). 
den Blick vermittelt, sondern thematisiert auch Relevanzsetzungen in der laufenden Kommunikation (Goodwin $2000 \mathrm{c}$ ).

Zweitens sind Formen und Modalitäten der visuellen Praxis situiert, d.h. es handelt sich dabei um kontextsensitive Praktiken des Sehens und Zeigens, die in einem Umfeld vollzogen werden, das aktiv einbezogen und genutzt, mithin dadurch auch etabliert wird. Goodwin (1994, 1995; Goodwin / Goodwin 1996) hat zudem gezeigt, dass in verschiedenen Professionen (Architektur, Navigation, Luftverkehrskontrolle, Lehre und Forschung) eine wesentliche Beschäftigung darin besteht, visuelle Phänomene in einer Weise zu identifizieren und zu klassifizieren, dass sie als für die jeweiligen Professionen spezifische Wissensobjekte etabliert werden.

Drittens handelt es sich bei visuellen Praktiken um verteilte Praktiken: Sehen ist keine rein individuelle, sondern eine soziale Aktivität, an der oft mehrere Individuen und ihre epistemischen Gewohnheiten beteiligt sind. So hat Hutchins (1995) demonstriert, wie die visuellen Praktiken, die dazu nötig sind, ein Schiff zu steuern, nicht in den mentalen Wissensvorräten einzelner Individuen gespeichert, sondern in ein verteiltes System eingebettet sind, das visuelle Geräte und Werkzeuge (Karten, Kompasse, Radarsysteme) ebenso umfasst wie unterschiedliche Akteure an verschiedenen strukturellen Positionen, die mit verschiedenen Hilfsmitteln je unterschiedliche visuelle Operationen durchführen. Erst im Zusammenspiel kann die Navigation des Flugzeugträgers erfolgen (vgl. Goodwin 2000 b: 163).

Viertens ist Sehen verkörpert, so dass es sich bei visuellen Praktiken insgesamt um reflexive, situierte, verteilte und verkörperte Praktiken handelt. Goodwin (1994) zeigt dies am Beispiel der skopischen Arbeit von Archäologen, die gemeinsam die Farbe von Erde auf einer Skala einschätzen. Dabei interagiert die verkörperte räumliche Organisation der Tätigkeit (die verkörperte Einnahme von Perspektiven, das Arrangieren von Instrumenten wie Lupen und Skalen, welche die Sichtbarkeit herstellen, etc.) mit der sequenziellen verbalen Aktivität. Der Fokus liegt hierbei also nicht auf visuellen Repräsentationen als isolierten Einheiten, sondern darauf, wie sie gemeinsam hergestellt und in fortlaufende Praktiken integriert werden (Goodwin 2000 b: 171).

\section{Sichtbarkeitsregime und visuelle Praktiken im Boxen}

Als Beispiel für eine im Rahmen verschiedener skopischer Regime und mit unterschiedlichen multimodalen Praktiken operierende Subkultur, durch die eine visuelle Konstruktion von Sozialität vollzogen wird, wird im Folgenden das Boxen untersucht. Boxen ist, wie die Analyse zeigen wird, ein Beispiel dafür, dass auch in einem sehr stark körperlich geprägten Bereich des Sozialen in unerwarteter Weise visuelle Praktiken und Konstruktionen am Werk sind und mithin eine skopische Subkultur vorhanden ist.

Um die durch diese Praktiken erzeugten visuellen Regime im Boxen zu identifizieren, werden im Folgenden Videoaufzeichnungen, ${ }^{4}$ die im Rahmen des an der Universität Bielefeld

4 Für die Darstellung in den Transkripten wurden die Videostandbilder mit Hilfe eines Computerprograms nachgezeichnet. Dies geschieht einerseits zum Zwecke der Anonymisierung der Beteiligten. Es bietet aber auch die Möglichkeit, das umfangreiche und für mit der Situation nicht vertraute Leser möglicherweise unübersichtliche Setting auf die (im Sinne der hier angefertigten Analysen) wesentlichrelevanten Aspekte zu konzentrieren. Soweit dadurch visuelle Informationen verloren gehen, welche für hier aufgestellten Analysen von Bedeutung sind (an einigen Stellen etwa die Blickrichtung der Beteiligten), ist dies im beschreibenden Text nach dem Transkript aufgeführt oder innerhalb eines Standbildes mit einem Pfeil gekennzeichnet. 
laufenden Forschungsprojektes „Kommunikation unter Druck“5 auf einem nationalen Meisterschaftswettkampf im Amateurboxen erstellt wurden, vor dem Hintergrund des hier skizzierten Ansatzes einer praxistheoretischen visuellen Soziologie mit den Instrumenten der ethnomethodologischen Konversationsanalyse analysiert. Die Analyse dieser Daten zeigt die Vielschichtigkeit und Vielgestaltigkeit visueller Praktiken im Boxen. Wie in allen Interaktionen, die unter der Bedingung der Kopräsenz stattfinden, laufen auch in den Interaktionen im und rund um den Boxring verschiedene visuelle Praktiken parallel. Diese Praktiken beziehen sich auf verschachtelte und sich überlagernde skopische Regime im Boxsport, die jeweils spezifische normative Erwartung an die Sichtbarmachung von Handlungen ebenso wie an deren Verdeckung stellen.

Eine erste reflexiv konstituierte Sichtbarmachung wird bereits bei der methodischen Herstellung des spezifischen „Realitätsakzents“ des Boxens vollzogen. Mit „Realitätsakzent“ bezeichnen wir, Schütz / Luckmann (2003: 55ff) folgend, das Repertoire an Mitteln, mit denen eine deutlich deviante soziale Praxis als unhinterfragte, unproblematische und selbstverständliche - d.h. lebensweltliche - Wirklichkeit, als sogenannte „Sinnprovinz“, etabliert wird.

Verhaltensweisen, die ansonsten sozial abgelehnt und sanktioniert würden - physische Gewalt gegen das Gegenüber insbesondere - sind im Boxen erwünscht und werden gefördert. Diese offensichtliche Spannung zwischen der alltäglichen und außeralltäglichen Welt wird durch rituelle Praktiken vermittelt. Ein Boxkampf wird in Bezug auf mehrere Dimensionen gerahmt und von der Alltagswelt abgegrenzt. So wird ein temporär und räumlich begrenzter Ort für die Austragung einer physischen Auseinandersetzung im sportlichen Wettbewerb geschaffen. Diese Rahmung erfolgt durch verschiedene gegenständliche wie performative Grenzziehungen. In Bezug auf die temporale Dimension dient die (zwar sichtbare, sicherlich aber besser hörbare) Glocke dazu, den Anfang und das Ende der Zeiträume zu markieren, in denen Gewalt erlaubt ist, während es außerhalb dieser Zeiten sanktioniert wird. Jedoch gibt der Glockenschlag immer nur die Rundenzeit an, das eigentliche Kommando zum Beginn des Kampfes kommt vom Ringrichter - indem er verbal „box “6 sagt und gestisch seine Hände wie eine Schranke zwischen die Kämpfer hält und dann zurückzieht. Die Geste bezieht sich auch auf ein räumliches Feld - den Ring -, der visuell markiert und als derjenige Raum herausgehoben wird, in dem Gewalt legitimiert und erlaubt ist, während sie außerhalb dieses Raumes verboten ist. Was die Dimension der Handlungsmacht (agency) anbetrifft, so zeigt sich, dass auch hier besondere Bedingungen herrschen: Es werden spezifische soziale Rollen und soziale Regeln etabliert, die nur für den Zeitraum und Ort des Kampfes gelten. Während des Kampfes müssen die Kontrahenten und auch alle anderen Beteiligten wie Trainer, Ringrichter, Punktrichter, Ringarzt, Zuschauer, Medien (und auch Forscher) diesen klaren, vorab definierten Regeln folgen. All ihre Aktivitäten werden in Bezug auf die Gefahr der Regelverletzung von eigens dafür benannten Rollenträgern beobachtet und evtl. sanktioniert. Einige der Beteiligten - besonders die Richter und Kontrollinstanzen der Sportorganisationen - werden rituell eingeführt,

5 „Kommunikation unter Druck: Praktiken der Verständigung von Trainern und Athleten im Spitzensport“, finanziert von der DFG, Laufzeit 2011-2015, Projektleiter: Jörg Bergmann, Carmen Borggrefe, Klaus Cachay und Christian Meyer, Fakultäten für Psychologie und Sportwissenschaft und für Soziologie, Universität Bielefeld. Die Aufnahmen wurden von Ulrich v. Wedelstaedt erstellt und von Eva Fenn und Julia Letetzki transkribiert, denen wir an dieser Stelle herzlich danken. Besonders danken möchten wir Eva Fenn für die Herstellung der Standbilder. Die Kämpfe stammen aus unterschiedlichen Gewichtsklassen, dauern aber alle jeweils drei Runden. Die Namen der Boxer sind anonymisiert. Im Rahmen des Projekts wurden mehrere Boxtrainer und die von ihnen betreuten Sportler begleitet und Videoaufnahmen von Training und Wettkämpfen angefertigt, jedoch immer nur ein Trainer bzw. Sportler zur gleichen Zeit. Daraus ergibt sich in der Kampfsituation die besondere Betonung der Aktivitäten einer der beiden Sportler - entweder des roten oder des blauen Boxers - und des jeweiligen Trainers.

6 Alle Kommandos des Ringrichters werden in englischer Sprache gegeben. 
indem sie durch den Hallensprecher benannt werden. Sie sind von da an - visuell sichtbar durch die Sitz- und Kleiderordnung sowie durch spezielle Insignien (Badges etc.) - mit bestimmten Rechten ausgestattet wie z.B. dem Recht, als vermittelnde und schiedsrichternde dritte Partei zu intervenieren und Regelverstöße zu bestrafen.

\subsection{Vorstellung und Symmetrisierung im Raum}

Um eine solche Redefinition und Redimensionalisierung der Lebenswelt zu erreichen, genügt es allerdings nicht, die Umdefinition und Neurahmung einfach durch einen Willensakt festzustellen oder auch zu proklamieren. Die neue Situationsdefinition (oder Sinnprovinz) muss vielmehr für alle Beteiligten augenscheinlich gemacht werden - beobachtbar, greifbar, erfahrbar und eben auch sichtbar - damit ihr Realitätsakzent modifiziert ist.

In Transkript 1 wird deutlich, ${ }^{7}$ wie die Redimensionalisierung des Gewöhnlichen hin zum Außergewöhnlichen im Wesentlichen durch ein Zusammenspiel hörbarer mit sichtbaren Komponenten körperlicher Aktivität - also praktisch - hervorgebracht wird. Während der Hallensprecher die Punktrichter, den Ringrichter und die Boxer namentlich vorstellt, positioniert der Ringrichter sie zugleich als - gerade auch in Bezug auf ihn selbst - Gleiche im Raum. Dies tut er durch eine fortlaufende Adjustierung seiner Gesten. Die Gegner werden nicht nur symmetrisch im Raum positioniert, sondern gerade auch in Bezug auf den unparteiischen Ringrichter. Die Symmetrisierung der Kontrahenten als Gleiche in Bezug auf das Regelwerk war zuvor schon mit dem Ritual des Wiegens sowie der damit verbundenen Einteilung in dieselbe Gewichtsklasse eingeleitet worden und wird jetzt fortgesetzt. Zugleich werden die Boxer für das Publikum, aber auch für alle anderen Anwesenden (inklusive für sich selbst) räumlichvisuell als Kontrahenten einander gegenüber gestellt.

\section{Transkript 1}

1

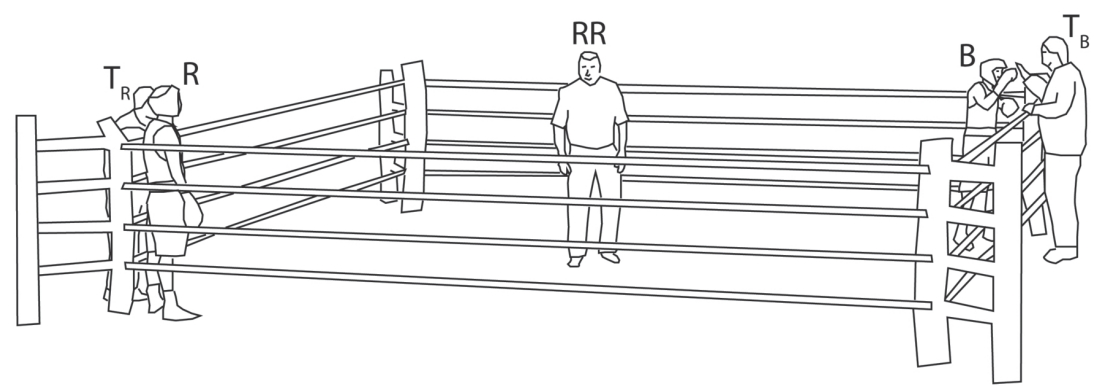

7 Weite Teile des erhobenen Materials wurden transkribiert und sequenziell analysiert. Aus Gründen des Umfangs und der Zugänglichkeit für nicht mit der Materie vertraute Leser muss jedoch das an dieser Stelle dargestellte multimodale Transkript weitaus selektiver ausfallen, als es bei konversationsanalytischen Verbaltranskripten gewöhnlich der Fall ist. 
2

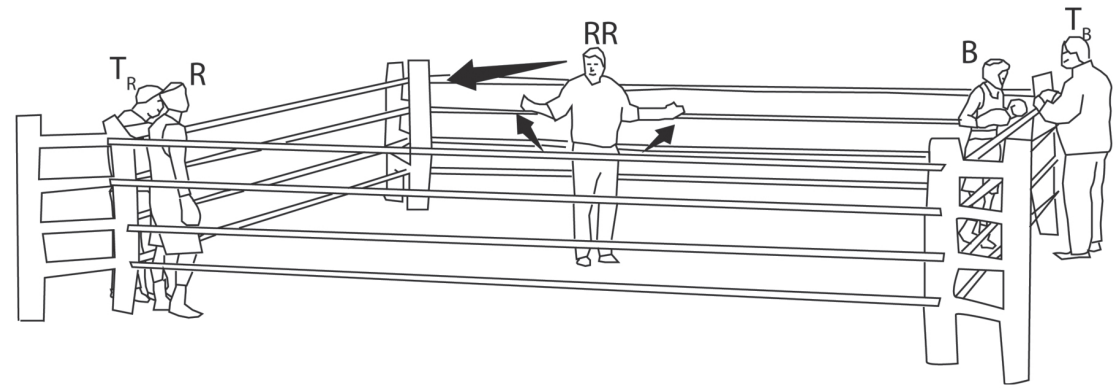

3

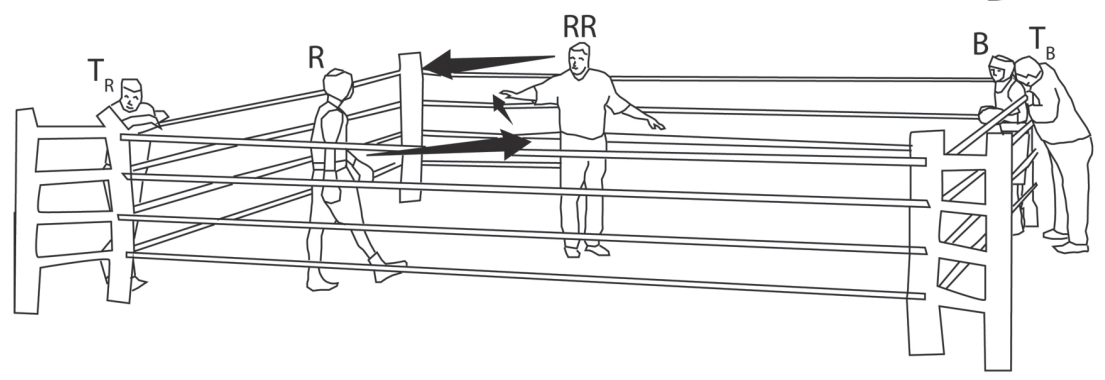

4

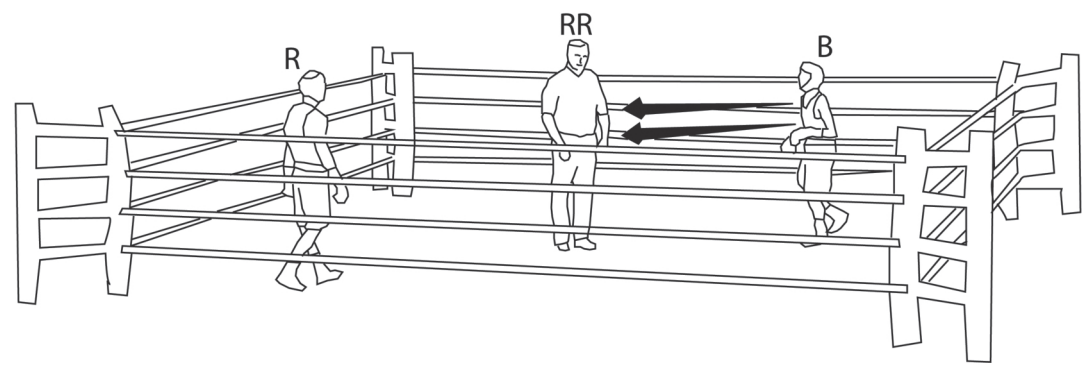

5
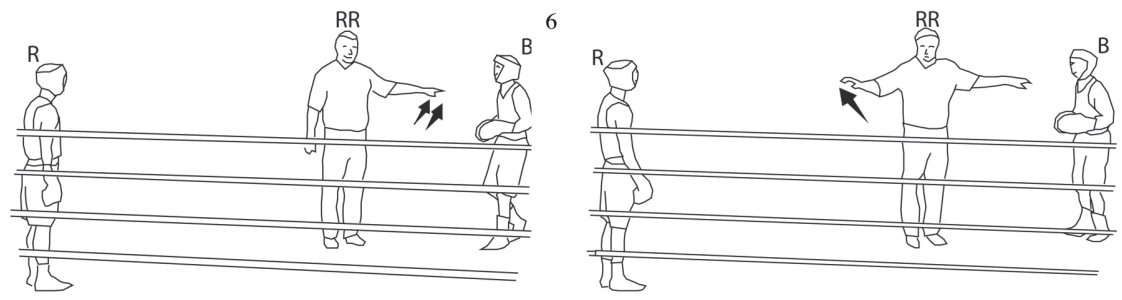
7
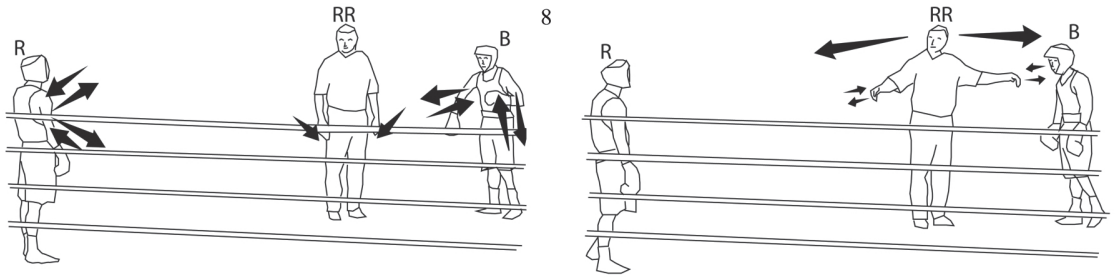

9

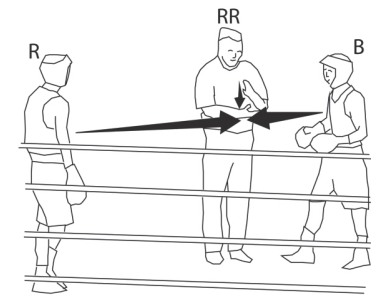

10

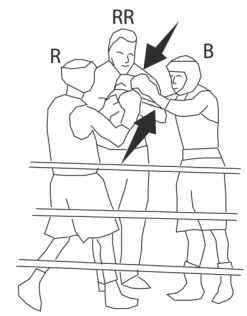

11

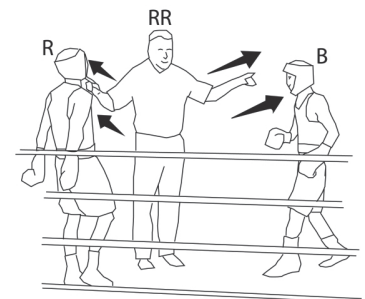

Quelle: eigene Daten

Wie man sieht, holt der Ringrichter die beiden Boxer in einer symmetrischen Handgeste zu sich (Standbild 2). Als der rechte, blaue Boxer (B, dem zum Zeitpunkt der Handgeste noch sein Mundschutz eingesetzt wird) zu schnell heranläuft (Standbild 4), bremst ihn der Ringrichter (Standbild 5), um einerseits die Synchronisierung mit der Vorstellung durch den Hallensprecher, andererseits aber zugleich die Äquidistanz in Bezug auf sich selbst zu wahren. Dann symmetrisiert er seine Geste noch einmal bezüglich des linken, roten Boxers $(\mathrm{R})$ und hebt die Hand in dessen Richtung (obwohl dieser sich bereits in einer Wartestellung befindet, Standbild 6), um seine Arme dann gleichmäßig zu senken (Standbild 7). Beide Boxer lockern ihre Muskeln (ebd.), bis der Ringrichter beide schließlich auf abgestimmte Weise und gleichzeitig zusammen vor sich holt und sich abklatschen lässt (Standbilder 8 bis 11; das eigentlich Kommando zum Boxen gibt der Ringrichter erst, nachdem er die Boxer wieder zurückgeschickt hat, also erst nach Standbild 11). Während des Vorgangs des Abklatschens passt er die Geschwindigkeit seiner Gesten an die Geschwindigkeit der Bewegungen der Boxer an. ${ }^{8}$

Während der Hallensprecher also alle am Kampf Beteiligten auditiv vorstellt, nutzt der Ringrichter den gleichen Moment, um - gewissermaßen in einer zweiten Ebene der gleichen Aktivität - die Symmetrisierung der beiden Athleten ihm selbst gegenüber sowie ihren Antagonismus sichtbar und praktisch hervorzubringen und dadurch zugleich als für sie und das Publikum evident zu symbolisieren.

Die große Bedeutung der Symmetrisierung ergibt sich aus dem hohen Grad der Abweichung der anschließenden Interaktion vom alltäglichen menschlichen Zusammenleben. Der Interaktionseröffnung kommt eine entscheidende Bedeutung für die Überführung der sozialen Situation von ihrer Unwahrscheinlichkeit - in diesem Fall einer physischen Auseinandersetzung der Beteiligten - hin zu einer für alle Anwesenden plausiblen Sinnprovinz zu (vgl. auch Hausendorf / Schmitt 2010: 55). Aufgrund des durch körperliche Gewalt hohen Abweichungsgrades muss im Boxen eine besonders intensive Umformung der Situation erfolgen. Durch die Herstellung der Gleichheit der Gegner vor dem Kampf, die durch eine aufwendige gestische Steuerung der Kämpfer erreicht wird, stellt der Ringrichter die Voraussetzungen für die soziale Akzeptanz des Kampfes her. Er visualisiert die Verlässlichkeit und Erwartbarkeit der nach-

8 Die Doppelpfeile symbolisieren eine höhere Geschwindigkeit von Bewegungen als einfache Pfeile. 
folgenden sozialen Situation für die Kämpfer, die anwesenden Trainer und Betreuer sowie das Publikum.

Eine solche Konstellation, in der unterschiedlich adressierte Sichtbarkeiten durch die körperlichen Praktiken aller am Kampf Beteiligten kollaborativ, aber auch antagonistisch erzeugt werden, charakterisiert grundsätzlich das soziale Geschehen „Boxen“. Zur Analyse dieser Überlagerung und Aufschichtung skopischer Praktiken ist oft die dritte und letzte Runde des Kampfes besonders interessant. In den Endphasen von Kämpfen spitzt sich aufgrund der Dramatik die Konkurrenz um die Sichtbarmachung spezifisch sportlicher sozialer Prozesse zu und verdichtet sich die visuelle Praxis der Beteiligten. Ein Teil der folgenden Beispiele stammt aus der dritten Kampfrunde.

\subsection{Die visuelle Performanz des Nichtgetroffenseins}

In Transkript 2 führt der blaue Boxer (B) nach zwei Runden mit einem Vorsprung von $13 \mathrm{zu}$ sieben Punkten ${ }^{9}$ und er versucht, diesen Vorsprung über die Zeit zu retten.

Transkript 2 (T: Trainer, A: Assistent) ${ }^{10}$
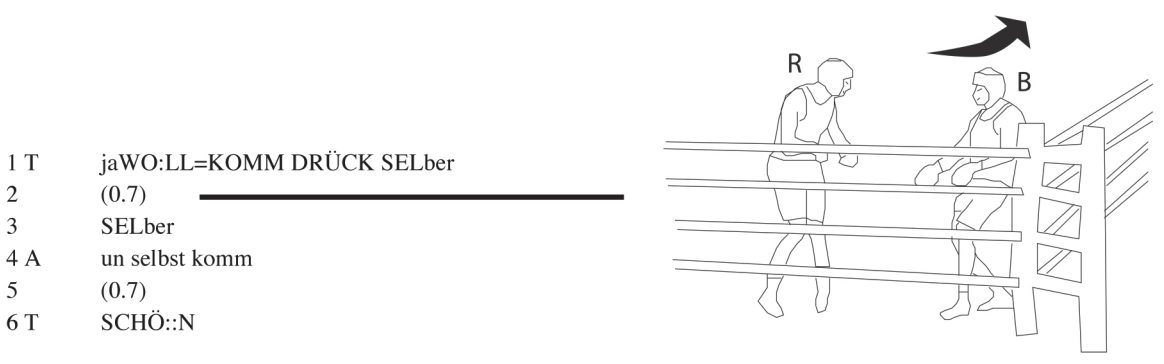

Quelle: eigene Daten

Der blaue Boxer (Zeile 2) weicht in dieser Szene entgegen den Aufforderung des Trainers (Zeilen 1,3) und Assistenten (Zeile 4) nach hinten aus, bis er an den Ringseilen steht. Dann dreht er sich um die eigene Achse nach links und weicht weiter zurück (Standbild, Zeile 2), indem er das Gewicht weit nach hinten verlagert und die Hände nach vorne nimmt. Im direkten Nahkampfgeschehen gibt er so seine Verteidigungshaltung auf - Oberkörper und Kopf sind nicht mehr vor Schlägen geschützt. Mit dieser riskanten Geste kann er jedoch anzeigen, dass er-obwohl ein körperlicher Nahkontakt stattgefunden hat - von seinem Gegner nicht getroffen wurde und weiterhin in der Lage ist, agil und leichtfüßig zu agieren. Diese Inszenierung ist nicht nur an das Publikum, sondern vor allem an die Punktrichter gerichtet, die durch Drücken eines Schalters einen Schlag als Treffer werten. Wenn innerhalb einer Sekunde mindestens drei (von fünf) Punktrichtern die Taste des gleichen Boxers gedrückt haben, wird diesem ein Punkt zurechnet. Aufgrund der unterschiedlichen Perspektiven, welche die einzelnen Punktrichter auf den Ring haben, ist es für den Boxer von grundlegender Bedeutung, deutlich - und idealerweise in mehrere Richtungen - anzuzeigen, dass er nicht getroffen wurde. Diese Sichtbarkeitspraxis des Boxers wird durch den Trainer daher mit einem auffällig langgezogenen und stark betonten ,Schö::n!' kommentiert (Zeile 6).

9 Die Wertung der Runde wird Publikum und Trainern am Rundenende auf Bildschirmen angezeigt.

10 Eine Aufstellung der Transkriptionssymbole findet sich im Anhang zu diesem Text. 
Auch Transkript 3 zeigt, wie einer der Boxer ostentativ gegenüber den Punktrichtern deutlich macht, dass die Aktion des Gegners kein Treffer war. Der Trainer ruft währenddessen konstant Anweisungen in den Ring.

\section{Transkript 3 (T: Trainer)}

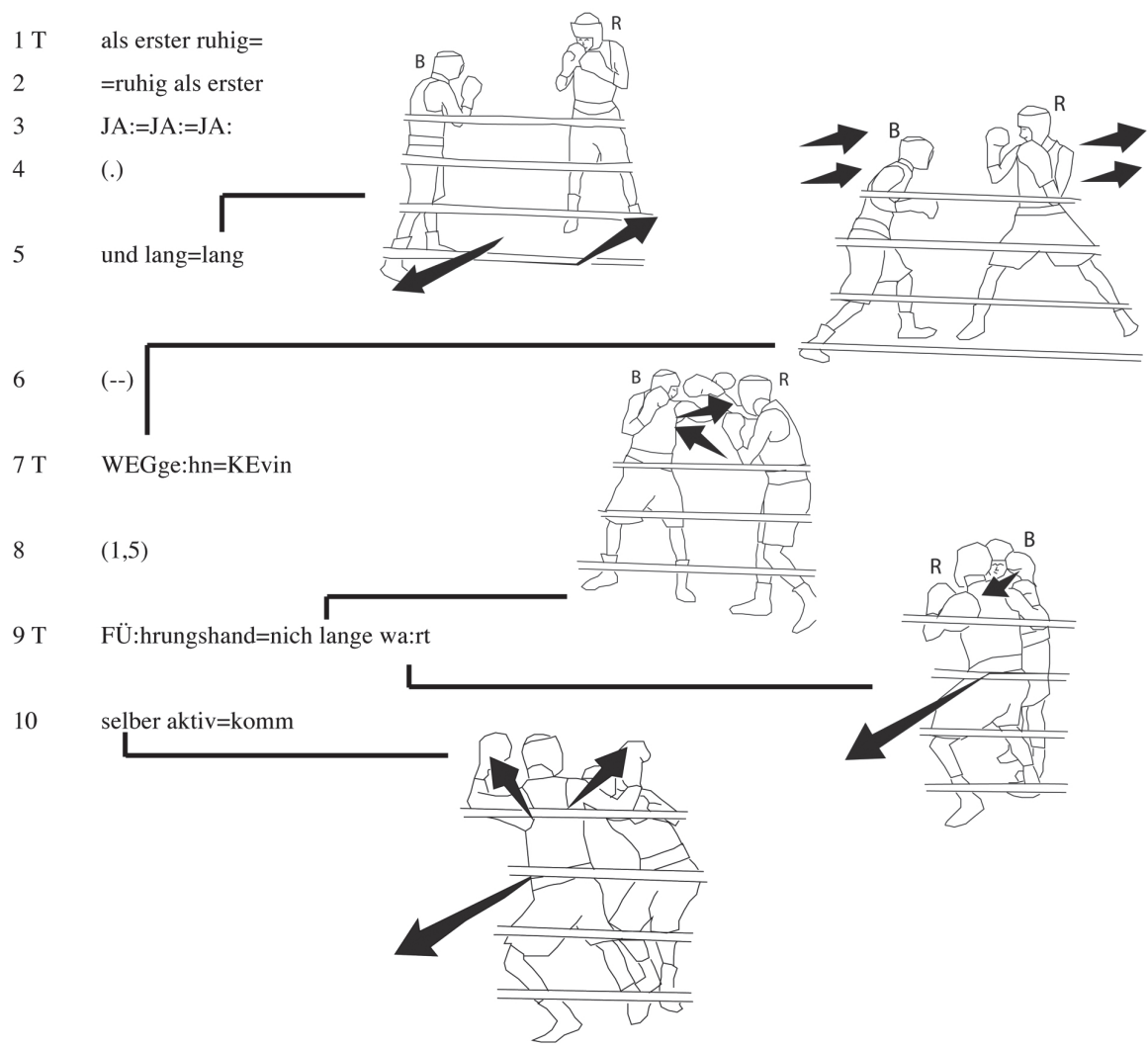

Quelle: eigene Daten

Nachdem beide Boxer zunächst voreinander tänzeln (Zeilen 1 bis 6), greift der blaue Boxer (B) an. In einer symmetrischen Wegbewegung gelingt es dem roten Boxer (R), den Abstand aufrechtzuerhalten und einen Treffer zu vermeiden (Zeile 7). Bei einem erneuten Angriff des blauen Boxers (Zeile 9) wird ein kritischer Abstand zwischen den Kontrahenten unterschritten, wodurch der rote Boxer gezwungen wird, zu demonstrieren, dass er nicht getroffen wurde allerdings in einer Weise, dass die Demonstration als natürlich und scheinbar beiläufig erscheint.

Auf derartige visuelle Inszenierungen des Nichtgetroffenseins wird häufig dann zurückgegriffen, wenn ein gewisser Abstand zwischen den beiden Kämpfern unterschritten wurde. Selbst wenn also Treffer gelandet wurden, können sie durch spezifische visuelle Praktiken zumindest für die Listen der Punktrichter ungeschehen gemacht werden. In diesem Sinne kommt der durch die Boxer und ihren Sichtbarkeitspraktiken hergestellten accountability bezüglich des Getroffenseins eine ebenso entscheidende Bedeutung für den Boxkampf zu wie 
einem tatsächlichen Treffer, der den Kontrahenten trifft und in seiner Körperlichkeit so beeinflusst, dass ihm (zumindest für einen Moment) seine Inszenierungsfähigkeit genommen wird.

\subsection{Die visuelle Performanz von Regelkonformität}

Eine zweite Schicht der Sichtbarkeitspraxis dient der ostentativen Performanz (der accountable-Machung) von Regelkonformität gegenüber dem Ringrichter. Nach einem Sturz des blauen Boxers (siehe weiter unten Transkript 7) kann der Kampf zunächst nicht fortgesetzt werden, da dessen Handschuh beim Sturz beschädigt wurde. Wird die Kampffähigkeit des Athleten nicht innerhalb von einer Minute wiederhergestellt, wird der Kampf beendet und entweder wiederholt oder zugunsten des gegnerischen Boxers gewertet. Aus diesem Grund wurde der Assistent losgeschickt, um einen Ersatzhandschuh zu besorgen. Das folgende Transkript 4 zeigt die dadurch entstandene Pause.

Transkript 4 (RR: Ringrichter, B: blauer Boxer)

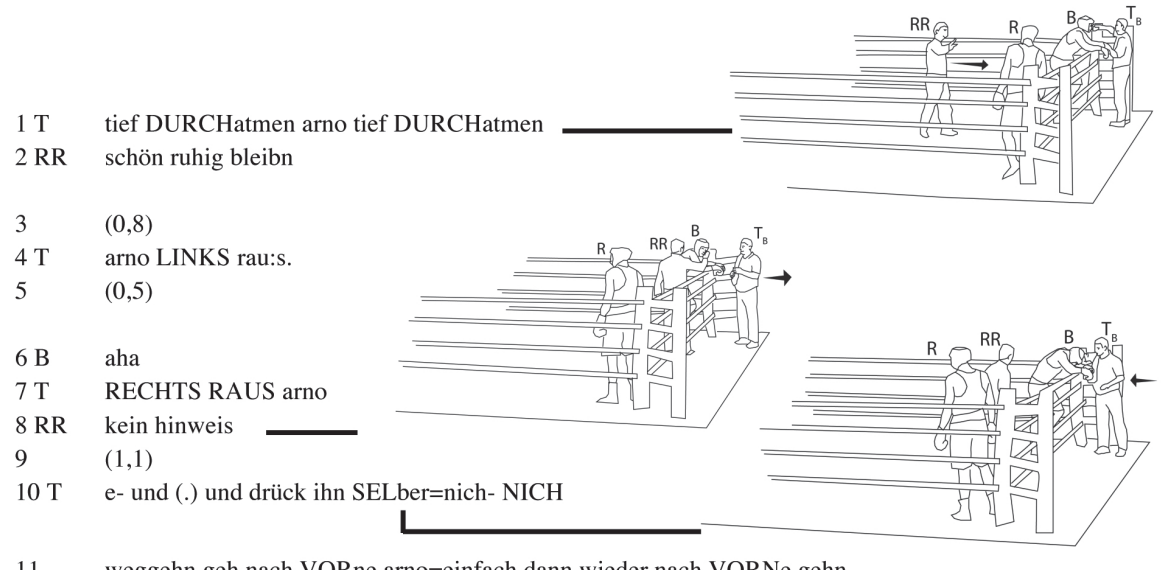

11 weggehn geh nach VORne arno=einfach dann wieder nach VORNe gehn

$12(0,4)$

ne? (.) wenn er sich LÖST, gehste hinterHER=tief

14 DURCHatmen. tief=mach dich GROSS

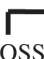

Quelle: eigene Daten

Der Ringrichter stellt zunächst den roten Boxer (R, Mitte) mit einer Handgeste in die neutrale weiße Ecke und fordert ihn auf, ruhig zu bleiben (Zeilen 1 und 2). Der Trainer $\left(T_{B}\right.$, rechts außen) des blauen Boxers (rechts im Ring, B) beginnt, nachdem er den eingerissenen Handschuh an seinen Assistenten gegeben hat, unmittelbar mit der Betreuung seines Boxers - er verteilt Wasser auf seinem Rücken (Zeile 1) und wendet sich verbal an ihn (Zeilen 1, 4, 7). Dies veranlasst den Ringrichter, den Trainer gestisch und verbal darauf hinzuweisen, keine 
Hinweise zu geben (Zeile 8). ${ }^{11}$ Der Trainer bewegt sich bereits beim Anblick des auf ihn zugehenden Ringrichters etwas von seinem Boxer weg (Zeile 8). Nachdem der Ringrichter sich wieder einen Schritt in Richtung des roten Boxers bewegt hat, berührt der Trainer seinen Boxer erneut und spricht wieder mit ihm (Zeile 10). Der Trainer führt hierbei allerdings jetzt eine Pendelbewegung durch, bei der er, immer wenn er in der Nähe seines Boxer ist, diesen berührt. Der Ringrichter und der rote Boxer blicken sich derweil nach dem Assistenten um, der den Ersatzhandschuh bringen soll. Die Ermahnung des Ringrichters führt hier zu keinem vollkommen veränderten Verhalten des Trainers. Er trägt der Ermahnung allerdings mit einer modifizierten visuellen Praxis Rechnung, indem er den Abstand zwischen sich und dem Boxer immer wieder vergrößert. Dieses Verhalten ändert er auch nicht, als der Ringrichter sich zu ihm umdreht. Insofern wird hier seine Orientierung an „Rezipienten“ (vgl. Sacks / Schegloff / Jefferson 1974) im Publikum sowie den anderen anwesenden Offiziellen deutlich.

Eine Aktivität, mit der ständig die ostentative Performanz von Regelkonformität gegenüber Ringrichter und Offiziellen dargestellt wird, ist das sogenannte Klammern oder Halten. Es bietet eine Möglichkeit für die Boxer, sich einige Momente der Ruhe vor Schlägen des Gegners zu verschaffen, indem sie sich mit den Armen umgreifen. Dies stellt jedoch einen Regelverstoß dar, der - je nach Ermessen des Ringrichters - lediglich mit einer Ermahnung, aber auch einer Verwarnung (die wiederum eine Punktewertung für den Gegner bedeutet) oder sogar einer Disqualifikation geahndet werden kann. Für den Boxer ist es gefährlich, wenn sein Gegner sich plötzlich löst und einen Schlag aus kurzer Distanz durchführt. Nachteilig ist auch, dass die Punktrichter nur schwer sehen können, was zwischen den beiden umklammerten Boxern passiert. Die Möglichkeit, Treffen bzw. Nichtgetroffensein erkennbar zu machen (Transkripte 2 und 3), ist also limitiert. Weiterhin gilt es, den Gegner als Initiatoren des Klammerns darzustellen. Trotzdem ist das Klammern weit verbreitet und kommt vor allem gegen Ende der Kämpfe immer häufiger vor. In der Regel führt es nur zu einer Unterbrechung des Kampfes (Kommando ,break' und eine konsequenzlose Ermahnung an mindestens einen der Boxer) und der anschließenden Wiederfreigabe durch den Ringrichter.

\section{Transkript 5}

1

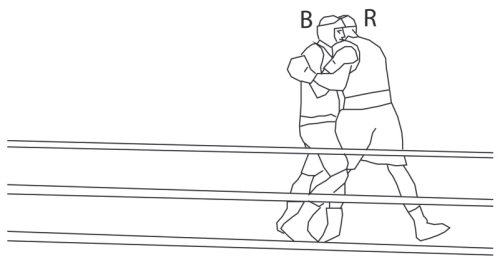

Quelle: eigene Daten
2

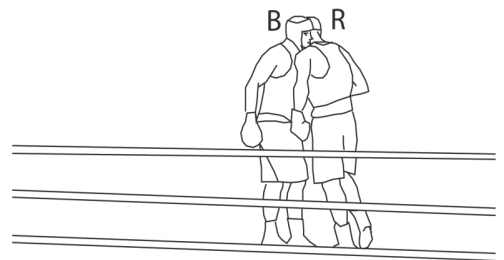

Während die Boxer in Standbild 1 noch eng beieinanderstehen, müssen sie nach der Anweisung des Ringrichters (, break') auseinandergehen. Jedoch verharren beide noch einen Moment, indem sie den Kopf auf die Schulter des Gegners gestützt und ihre Körper gegeneinander gelehnt halten (Standbild 2). Die Boxer bewegen sich hier im Spannungsfeld zwischen wertvollem Ausruhen und den Gefahren des Nahkontakts zum Gegner bzw. des Regelverstoßes, der bei knappen Punktentscheidungen ausschlaggebend für Sieg oder Niederlage sein kann.

11 Das Regelwerk bleibt bezüglich eines Verbots, in einer solchen Situation Hinweise oder Hilfestellungen geben zu dürfen, allerdings uneindeutig. Geregelt ist lediglich, dass nur der Sekundant (Trainer) das Ringpodium - nicht aber den Ring - betreten darf. 
Das Herunternehmen der Arme führt dem Ringrichter, den Punktrichtern und dem Publikum das Ende des Klammerns vor, womit jeder der beiden anzeigt, dass er nicht der für das Klammern verantwortliche Boxer ist. Hierdurch signalisieren sie einerseits Regelkonformität und andererseits auch körperliche Stärke (dass sie das Klammern zum Ausruhen nicht nötig haben). Das Herunternehmen der Hände macht sie jedoch zugleich angreifbar für den Gegner. Schließlich spricht der Ringrichter eine Ermahnung aus und setzt den Kampf fort. Die visuell orientierten körperlichen Aktivitäten und Gesten der Boxer im Klammern richten sich also simultan an verschiedene skopische Regime, die von Ringrichtern und Offiziellen, Gegnern oder dem Publikum ausgeübt werden.

\subsection{Die visuelle Performanz von Vitalität und Selbstkontrolle}

Ein drittes Sichtbarkeitsregime, das bereits angesprochen wurde, besteht in der Notwendigkeit zur Demonstration körperlicher Stärke. Nachdem der Trainer noch einmal taktische Anweisungen an den Boxer gegeben (siehe oben Transkript 4, Zeile 13) und ihn erneut zum tiefen Durchatmen aufgefordert hat, bedeutet er ihm, sich groß zu machen (siehe oben Transkript 4, Zeile 14).

Transkript 6 (Auszug aus Transkript 4, Zeile 12ff.; T: Trainer)
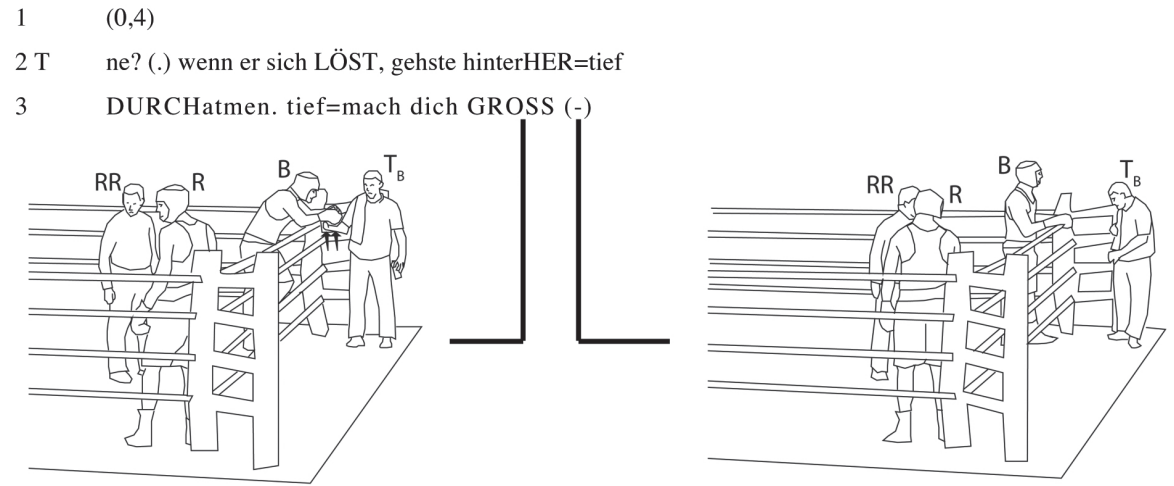

Quelle: eigene Daten

Gestisch begleitet er dies mit einem Schlag auf die Unterseite des (verbliebenen) Handschuhs. Durch diese Geste zeigt er an, dass seine verbale Anweisung nicht auf die Fortsetzung des Kampfes, sondern auf den unmittelbaren Moment gerichtet ist. Die Orientierung an Punktrichtern und vor allem am Publikum und deren unterstellter Wahrnehmung der Körperlichkeit des Boxers ist evident: das höchste Maß körperliche Regeneration könnte der Boxer durch Sitzen erlangen, jedoch darf der vorhandene Sitz nur in den Rundenpausen in den Ring geklappt werden. Aber auch das Lehnen auf die Seile wird ihm durch den Trainer verwehrt. In der Situation der Kampfunterbrechung dient die Körperhaltung des Boxers einzig zur Inszenierung seiner Körperlichkeit. Der Boxer reagiert auf die Aufforderung des Trainers umgehend: Das zweite Standbild (Zeile 3) zeigt seine veränderte Haltung.

Die Punktrichter sind ständig aufmerksam auf das Geschehen im Ring: sie schauen ununterbrochen auf die Boxer, und auch während Unterbrechungen, Rundenpausen oder der eigenen Punktevergabe per Knopfdruck wandert der Blick nicht vom Geschehen im Ring weg. Sie zählen Treffer und bewerten die Überlegenheit eines der beiden Boxer im Nahkampf. Von Boxer und Trainer wird ihnen unterstellt, bei der Verrichtung ihrer skopischen Tätigkeit un- 
unterbrochen nach visuellen Anhaltspunkten zu suchen, ähnlich zum „,coding [of the] events within a relevant perceptual field“ (Goodwin 1994: 619), das Goodwin für das professionelle Sehen von Polizisten beschrieben hat. So wie diese Körperbewegungen oder -haltungen von Verdächtigen als kooperativ oder aggressiv „,codieren“, nehmen Punktrichter nach Auffassung der Boxer und Trainer Codierungen im Hinblick auf die körperliche Stärke oder Schwäche der Boxer vor, die Einfluss auf ihre Beurteilung und letztlich die Punktezählung hat. Entscheidend ist daher die Fähigkeit des Boxers zur Selbstkontrolle, denn manche Schläge treffen so hart, dass ihm die Fähigkeit zur Inszenierung etwa des Nichtgetroffenseins kurz genommen wird und er sich träge bewegt, zu torkeln beginnt, sich nicht länger auf den Beinen halten kann und zu Boden geht. Das ,in den Seilen Hängen“ ist im Boxsport identisch mit einem K.O., denn wenn der Boxer in den Seilen hängt und diese nicht aus eigener Kraft verlassen kann, wird er angezählt. Die Inszenierung der Selbstkontrolle ist also, gerade wenn Kontakt zu den Seilen besteht, von größter Bedeutung.

Das nächste Beispiel zeigt die der Unterbrechung in Transkripten 4 und 6 vorangegangene Szene, in welcher der blaue Boxer bei einem erneuten Versuch, sich aus einer Position in der Nähe der Seile zu lösen, über das Bein seines Gegners stürzt. Neben dem ,Hängen in den Seilen' stellt der Sturz auf den Boden bzw. Niederschlag den anderen wichtigsten Kontrapunkt für die Inszenierung der eigenen Selbstkontrolle durch den Boxer dar.

\section{Transkript 7 (A: Assistent, T: Trainer)}

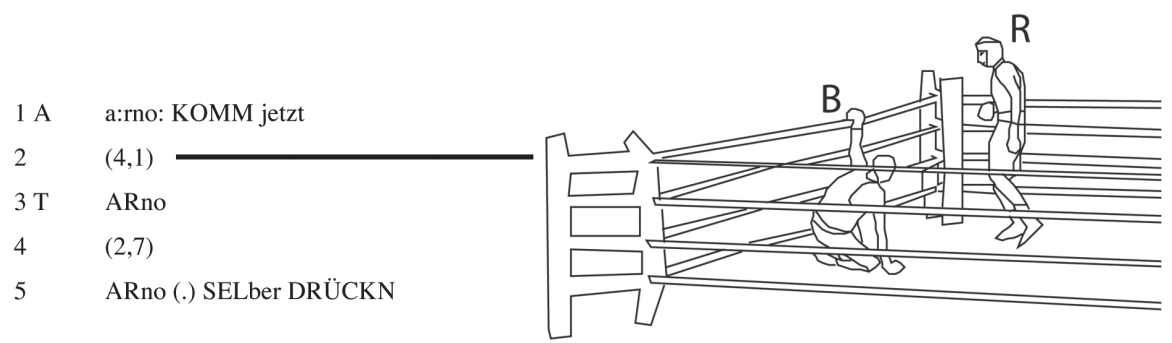

Quelle: eigene Daten

Der gestürzte Boxer steht nun vor der Herausforderung, sein Fallen als eines darzustellen, das weder einem Treffer noch körperlicher Entkräftung geschuldet ist, sondern einem zufälligen Stolpern. Anders als etwa im Fußball ist es ihm nicht möglich, an dieser Stelle auf die Involviertheit des Gegners in seinen Sturz zu verweisen, etwa indem er sich auf dem Boden rollt und sein Bein hält o.ä. Ein Verbleiben auf dem Boden würde zum einen nach dem Anzählen potenziell zur Beendigung des Kampfes führen. Zum anderen bedeutet jedes Zeichen körperlicher Schwäche einen Nachteil.

Obwohl sich der Boxer noch auf dem Boden befindet, setzt der Trainer (Zeile 3 und 5) seine Anweisungen an den Boxer in der gleichen Weise fort, wie er das auch während des Kampfes selbst tut. Er wirkt dadurch an der Inszenierung des Sturzes durch den Boxer als unproblematischen Gestolpertseins mit und widerspricht gleichzeitig mit ihm zusammen einer möglichen Interpretation des Sturzes als eines Hinweises auf körperliche Schwäche oder Getroffensein. Zwar hat ein solches Ereignis alleine noch keine unmittelbaren Konsequenzen für das Kampfgeschehen oder die Punktevergabe - erst wenn der Boxer mehrmals innerhalb einer Runde oder im gesamten Kampfverlauf zu Boden geht und angezählt wird, wird dies als Niederschlag (K.O.) gewertet, - doch die Beteiligten sind stets darum bemüht, einen Eindruck der körperlichen Stärke zu vermitteln, da sie diesem eine Wirkung auf die Punktevergabe und das Pu- 
blikum (und diesem wiederum eine Wirkung auf die Punktevergabe) zuschreiben. Ganz besondere Bedeutung erhält diese Zuschreibung von Stärke, Selbstkontrolle und Kontrolle der Kampfsituation $^{12}$ in einem sehr ausgeglichenen Kampf. Haben beide Kämpfer nach Kampfende die gleiche Punktzahl, entscheiden die Punktrichter per Vergabe eines Hilfspunkts über den Sieger. Die Punktrichter sind dabei nicht an ihre vorherige Wertung der Treffer gebunden (die ihnen auch nicht vorliegt), sondern entscheiden nach ihrem Gesamteindruck, wer der überlegene Boxer war. In diesen Situationen ist der Eindruck der Selbstkontrolle, den der Boxer bei den Punktrichtern hinterlassen hat, direkt entscheidend für den Ausgang des Kampfes. Neben dem oben erwähnten Wettkampf um die Sichtbarmachung des Nichtgetroffenseins beziehen sich daher andere visuelle Praktiken auf die Sichtbarmachung körperlicher Stärke und Vitalität gegenüber Gegner, Punktrichtern und dem Publikum.

\subsection{Die visuelle Performanz von Fairness}

Neben der Interaktion mit Trainer, Gegner, Ringrichter und Punktrichtern sind einige der visuellen Aktivitäten der Boxer direkt an das Publikum gerichtet. Einen solchen Fall stellt der Kampf dar, in dem der blaue Boxer zwar aufgrund eines Stolperns zu Boden gegangen war, aber dennoch gewonnen hatte. Nach der Übermittlung der Punktrichterentscheidung waren aus dem Publikum laute Buhrufe zu hören. Dies ist eher ungewöhnlich und wurde während der Forschung ansonsten nur in Fällen beobachtet, in denen regelwidrige Aktivitäten vorgekommen waren (z.B. ein Tiefschlag). Der blaue Boxer hatte aber durch die Unterbrechung gegenüber seinem Gegner einen Vorteil erlangt: Während der rote Boxer in die neutrale weiße Ecke gestellt wurde, konnte der blaue Boxer in seine eigene Ecke gehen und dort von seinem Trainer betreut werden.

Transkript 8 (T: Trainer)

1

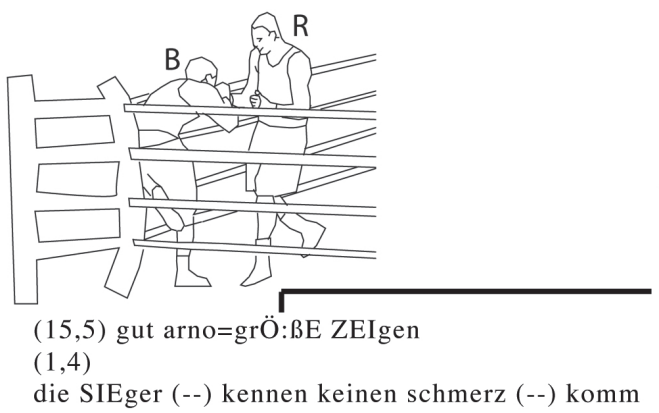

Quelle: eigene Daten

$3 \quad(1,4)$

$4 \mathrm{~T}$ die SIEger (--) kennen keinen schmerz (--) komm

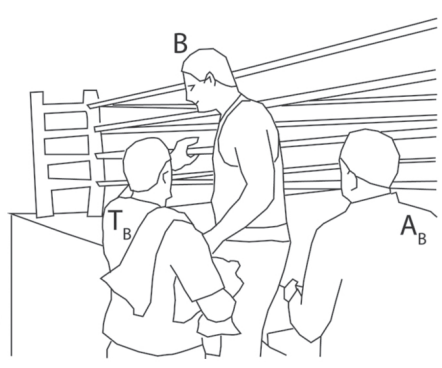

Nach der Verkündung der Entscheidung durch Hallensprecher und Ringrichter und dem obligatorischen Händeschütteln mit den gegnerischen Trainern und Betreuern hält der siegreiche blaue Boxer dem Verlierer die Seile auf. Dies ist eine hochgradig symbolische Handlung, vor allem, da die Seile so straff gespannt und schwer sind, dass sie sich durch diese Maßnahme in ihrer Position kaum verändern. Der rote Boxer tritt heran, gibt seinem Gegner noch einmal die Hand (Standbild 1) und klettert dann durch die Seile. Mit seiner Geste richtet

12 In vergleichbarer Weise versuchen die Prozessbeteiligten im Rodney King-Prozess laut Goodwin (1994, v.a.: 618; vgl. Goodwin / Goodwin 1997) unter Rückgriff auf visuelle Praktiken und eine durch diese etablierte Deutungshoheit die Frage zu klären, wer in welchen Momenten Einfluss auf einen eskalierenden oder deeskalierenden Fortgang der Prügelei durch die Polizei hat. 
sich der Sieger hier an den gegnerischen Boxer, aber auch an die Öffentlichkeit in der Halle, zu der auch viele andere an der Meisterschaft teilnehmende Boxer gehören. Nachdem der blaue Boxer ebenfalls vom Podium heruntergestiegen ist, ermuntert ihn sein Trainer demgegenüber - wiederum an Publikum und potenzielle zukünftige Gegner gerichtet - zum „Größe zeigen“: Sieger kennten ,keinen Schmerz“ (Zeilen 2 bis 4).

\subsection{Die Manipulation von Sichtbarkeiten}

Sichtbarkeit ist, wie wir bereits gesehen haben, keine objektive Tatsache, sondern dient der Manipulation der skopischen Praktiken von Publikum, Gegner, Ring- und Punktrichter sowie Offiziellen. Besonders in der letzten Kampfphase erlangt das Boxen immer stärker eine nicht nur verkörperte, sondern tatsächlich auch karnale, fleischliche Dimension. Verletzungen wie geschwollene Augen oder Cuts, die jetzt sichtbar werden, können von den Punktrichtern direkt als Zeichen für erfolgreiche Treffer des Gegners gewertet werden und so das Ergebnis beeinflussen. Die gewissermaßen ultimative Form der Sichtbarkeit für einen erfolgreichen Schlag ist sicher der K.O. Aber selbst in diesem Fall versucht der gefallene Boxer, schnell aufzustehen und zu demonstrieren, dass er nicht bewusstlos, sondern z.B. nur ausgerutscht oder gestolpert war (siehe oben Transkript 7).

Während die Boxer also einerseits ständig damit beschäftigt sind, die Legitimität und den Erfolg ihrer Aktionen nach außen sichtbar und verbuchbar zu machen, versuchen sie andererseits, ebenso viele andere Aspekte ihrer eigenen Aktionen (Absichten, Manöver, Taktiken) oder der Aktionen des Gegners (Treffer) zu verbergen und gegenüber dem Gegner, dem Ringrichter oder den Punktrichtern durch Finten, Verschleierung und Täuschung unsichtbar zu machen. Diese Täuschungsmittel basieren wie in vielen anderen Bereichen des Lebens auf der Existenz eines zunächst kooperativen, sozialen Sichtbarkeits-Prinzips.

Die Tatsache also, dass (wie so oft) Sichtbarkeit die einzige mögliche und verlässliche Quelle für die Bewertung der sozialen Situation Boxen seitens der Punktrichter ist, verstärkt die Notwendigkeit von Täuschungspraktiken. Diese sind besonders effektiv bei Aktionen wie erfolglosen Schlägen, die dennoch als Treffer verbucht werden, und erfolgreichen Treffern, die als Luftschläge gewertet werden.

Auch wenn Fleischwunden (Cuts) scheinbar unumstritten und unbestreitbar sind, können auch hier Täuschungsmanöver eine Rolle spielen. Trainer und Boxer versuchen daher durchaus, Verletzungen zu verbergen. Dies zeigt das nächste Beispiel, in dem der Trainer (links) und sein Assistent (rechts) in einer Rundenpause verdeckt Vaseline auf einen Cut am Auge des Boxers schmieren (Zeilen 1, 3, 7). ${ }^{13}$

13 Die Vaseline ist durch ein dickes schwarzes Kreuz gekennzeichnet. 
Transkript 9 (T: Trainer)

$1 \mathrm{~T}$ oh wat hattn DER=der hat Auge uff

2 ((Glocke läutet))

3 vaseLIne

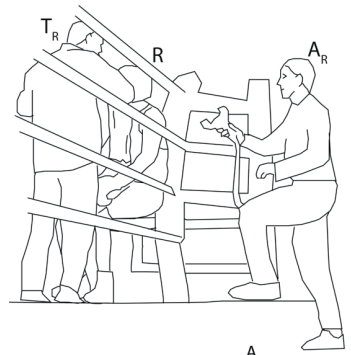

4 DURCHatmen sehr gut pass uff hast äh fünfe

$5 \quad$ VORne=tie:f durchatmen SO lass nich

6

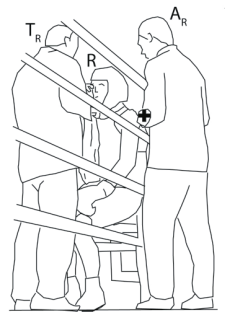

die FÜHRUNGShand oben=führungshand

9 versuch nochma höher zuhauen=

$10=$ schlägste die RECHte schlägst die RECHte

11 ja mein spross
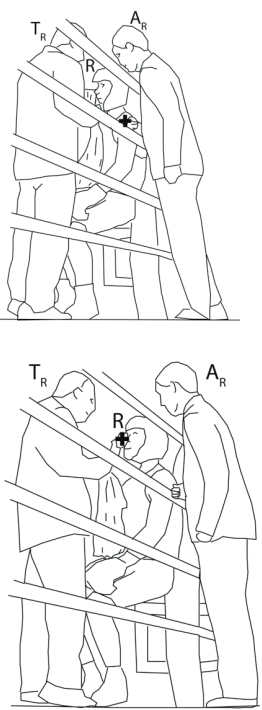

Quelle: eigene Daten

Materialitäten wie die Haut des Boxers können also zusätzlich zur Performanz eine Form von Sichtbarkeit herstellen. Sie können zu einem wichtigen Bestandteil des „Netzes bedeutsamer Sichtbarkeiten“ werden, über die letztlich die Punktrichter den Sieger und Verlierer des Kampfes festlegen. In ihrem manipulativen Bemühen versuchen die Beteiligten, ihre sichtbaren Praktiken so auszugestalten, dass sie von anderen gerade nicht als bedeutsam erachtet werden - im Sinne eines de-emphasizing als Gegensatz zum ,highlighting“ (Goodwin 1994: 609). 


\subsection{Verteilte visuelle und körperliche Praxis}

Die letzten Beispiele zeigen eine weitere Dimension von Visualität im Boxen, nämlich die Fähigkeit der Beteiligten, die Kampfsituation skopisch gemeinsam zu bewältigen. In diesem Fall ist dies vor allem die Unterstützung des Boxers durch die optische Wahrnehmungsfähigkeit des Trainers bzw. des Assistenten.

Transkript 10 (T: Trainer, A: Assistent)

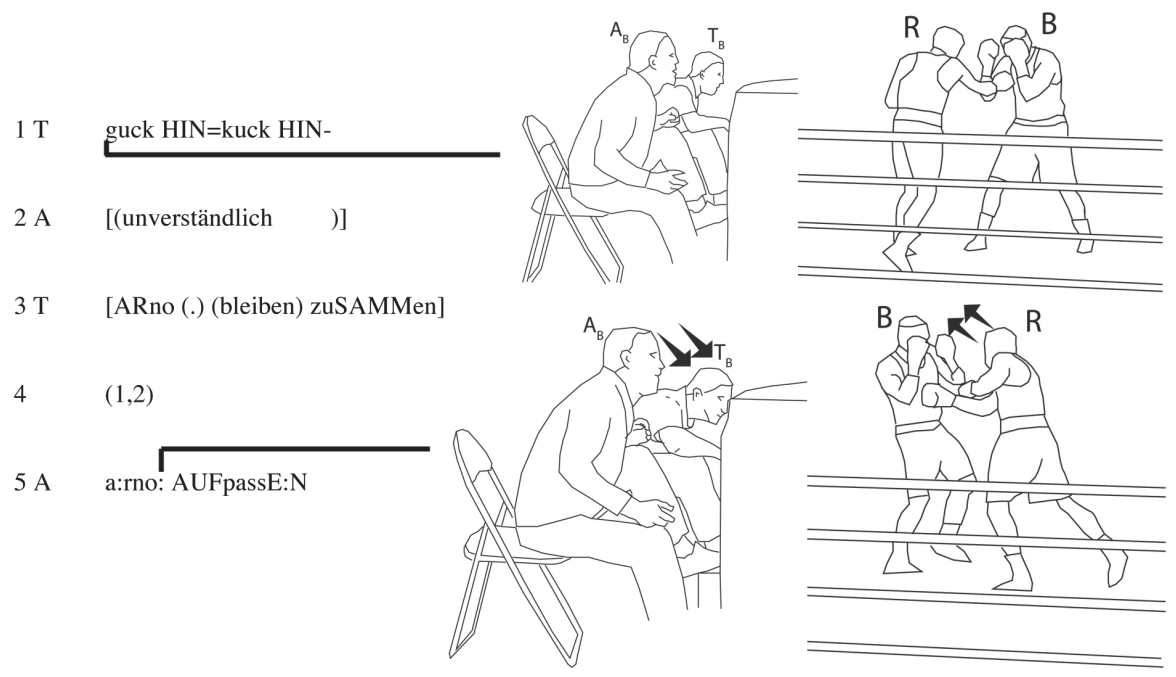

Quelle: eigene Daten

Trainer und Assistent antizipieren hier die Schläge des Gegners und warnen den Boxer vor ihnen. Sie stellen auf diese Weise ihre Wahrnehmungsfähigkeit gewissermaßen in den Dienst des Boxers und kommunizieren dem Sportler im Ring verbal ihre visuellen Wahrnehmungen. Den Trainern steht von außerhalb des Rings ein ganz anderer skopischer Raum zur Verfügung. Dies ergibt sich schon alleine aus dem Umstand, dass ihr Sichtfeld nicht permanent durch Schläge des Gegners und eigene Abwehrmaßnahmen eingeschränkt und ihre Fähigkeit zur kognitiven Verarbeitung des Gesehenen nicht durch Schläge gegen den eigenen Körper (vor allem auf den Kopf) negativ beeinflusst wird. Zudem kann den Trainern eine besonders hohe Expertise bezüglich des Erkennens taktischer Muster oder anderer Aspekte im Verhalten des Gegners zugestanden werden. Über die verbale Vermittlung wird so dem Boxer die speziell geschulte Beobachtungs- und Interpretationsgabe der Trainer zur Verfügung gestellt.

Besonders beim Trainer ist zu beobachten, dass er den Kampf auch körperlich mitvollzieht. Er duckt sich mimetisch und ,taucht', so wie es der Boxer tun sollte, um den Schlägen des Gegners auszuweichen (Zeile 5 sowie in Transkript 11, Zeile 4). Der Trainer ist der hauptverantwortliche und persönliche Coach des Boxers. Er trainiert nicht nur nahezu täglich mit ihm, er hat auch die langfristige (intensives Trainingslager in den letzten Wochen) und unmittelbare Vorbereitung auf den Kampf (in der Kabine) mit dem Boxer absolviert. Beim sogenannten ,Einschlagen' in der Kabine simuliert der Trainer zum einen den Gegner und lässt den Boxer in die Pratzen schlagen. Er gibt dem Boxer zum anderen aber auch verbal taktische Anweisungen, wie er zu schlagen und sich zu bewegen hat. Auf diese Weise stimmt er den Boxer auf seine Anweisungen ein, übt bestimmte Schlagrhythmen mit ihm ein und weist auf Besonder- 
heiten des Gegners hin. Boxer und Trainer adjustieren ihre Körper in diesem Prozess, die Stimme des Trainers wird mit den Körperbewegungen des Boxers abgestimmt. Der Trainer kann so während des Kampfes durch stark abgekürzte, schlagworthafte Anweisungen direkt das verkörperte Wissen des Boxers mit seiner Stimme abrufen, das er zuvor in jahrelangen Trainingseinheiten mit ihm erarbeitet hat. Dies wird im folgenden Ausschnitt erkennbar, der zeigt, dass sich der Blick des Trainers nicht durch nur durch verbale Kommunikation mit den Aktionen des Boxers verbindet, sondern die Beteiligten ein breites Spektrum multimodaler Ressourcen einsetzen.

Transkript 11 (T: Trainer)

$1 \mathrm{~T}$

HE::: ((klatscht 3 mal))
2

3

4

$$
(0,5)
$$

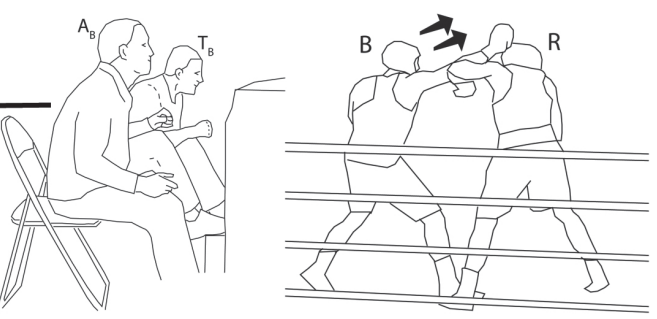

kuck HIN
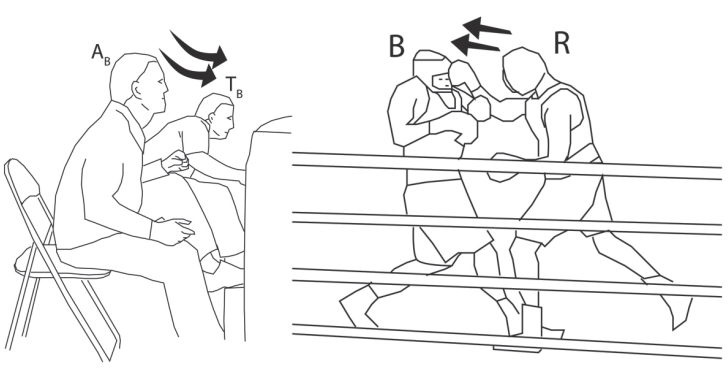

Quelle: eigene Daten

In Zeile 1 beschränken sich die verbalen Anweisungen des Trainers auf ein lang gezogenes und stark betontes ,he', worauf ein dreimaliges Klatschen folgt. Der Informationsgehalt dieser Äußerung scheint zunächst gering. Jedoch sind es diese und ähnliche Anweisungen, die in der Vorbereitungsphase auf den Kampf durch den Trainer immer wieder eingeübt werden, wenn er mit dem Boxer bestimmte Manöver ausführt. Das mehrfache Klatschen korrespondiert unmittelbar mit einem eingeübten Schlagrhythmus, den der Trainer hier beim Boxer abzurufen versucht, wodurch er den Boxer in seiner vorwärts gerichteten Angriffsbewegung auf den Gegner hin unterstützt. Durch dieses gestisch-akustische, paraverbale Äußerungsformat gelingt ihm erstens der Zugriff auf verkörpertes Wissen des Sportlers (bzw. auf verkörpertes Wissen, das von beiden geteilt wird) und zweitens die Verbindung seiner eigenen skopischen Praxis mit den körperlichen Aktivitäten des Boxers. Aufgrund der enormen Schnelligkeit, mit der sich das Kampfgeschehen permanent ändert, kann dies häufig nur mit solchen stark verkürzten, fast analog zu nennenden paraverbalen Beiträgen wie dem Klatschen geschehen.

Im weiteren unmittelbaren Verlauf dreht sich dann allerdings das Kampfgeschehen um und der blaue Boxer gerät in die Defensive. Deutlich sichtbar ist hier, dass der Trainer in dem Moment, als der Boxer getroffen wird, eine Ausweichbewegung nach unten links vollzieht (Zeile 4). Die Ausweichbewegung in diese Richtung wäre für den blauen Boxer sinnvoll gewesen, da der rote Boxer mit der Rechten schlägt. Erkennbar wird daran die mimetisch be- 
gründete Antizipationsleistung, die der Trainer in diesem Moment vollbracht hat. Augenblicke vor dem Schlag erkennt er diesen bereits und warnt seinen Boxer davor (Zeile 3) - wenngleich erfolglos. Mit Hinweisen wie ,guck hin' und , aufpassen' erweitert der Trainer so das Wahrnehmungsfeld des Boxers um die Perspektive des Trainers.

Die Vermittlung zwischen beiden Personen verläuft als ein multimodal organisierter Prozess, in dem zur Kommunikation der verteilten Skopizität zumindest auf Seiten des Trainers neben Verbalität auch auf paraverbale Äußerungsformate zurückgegriffen wird, um der besonderen Temporalstruktur der Interaktion im Boxring gerecht zu werden. Das Sehen verteilt sich somit im hier dargestellten Fall auf mindestens zwei oder - nimmt man den Assistenten dazu - drei Personen. Wie bereits bei Goodwin beschrieben, ist „the ability to see [...] lodged not in the individual mind" (1994: 633); vielmehr es kommt zu einem verteilten Sehen. Interessanterweise entsteht dabei eine Verteilung in beide Richtungen, denn letztlich wird auch der Trainer an die Wahrnehmungsfähigkeit des Boxers zurückgebunden: Aufgrund seiner Kenntnisse über das verkörperte Wissen des Boxers und indem er die in der Auseinandersetzung mit dem Gegner vollzogenen Bewegungen beobachtet, erkennt er visuell die Widerstände und Defizite, denen sich dieser ausgesetzt sieht. Er ist so auch in der Lage, die abnehmende Wahrnehmungsfähigkeit des Boxers - etwa aufgrund von zunehmender Erschöpfung - zu antizipieren und verstärkt zu substituieren (mittels seiner eigenen Wahrnehmung und der verbalen oder paraverbalen Kommunikation selbiger in den Boxring hinein).

Diese ,verteilte Agency“ kann durchaus dazu führen, dass der Boxer nicht weiter boxt (und Gefahr läuft, gegnerische Angriffe nicht abwehren zu können), wenn der Trainer seine skopischen Aufgaben nicht mehr erfüllt und keine Anweisungen mehr in den Ring ruft, wie das letzte Beispiel dieses Texts (siehe weiter unten Transkript 13) zeigt.

Die enge Beziehung zwischen Boxer und Trainer, wie sie die letzten beiden Beispiele dieses Texts beleuchten, wird vor allem durch die permanente Ansprache des Trainers aufrechterhalten. Im folgenden Transkript ist zunächst zu sehen, wie eng die vokalen Beiträge des Trainers und die Aktionen des Boxers im Idealfall korrespondieren.

Transkript 12 (T: Trainer)

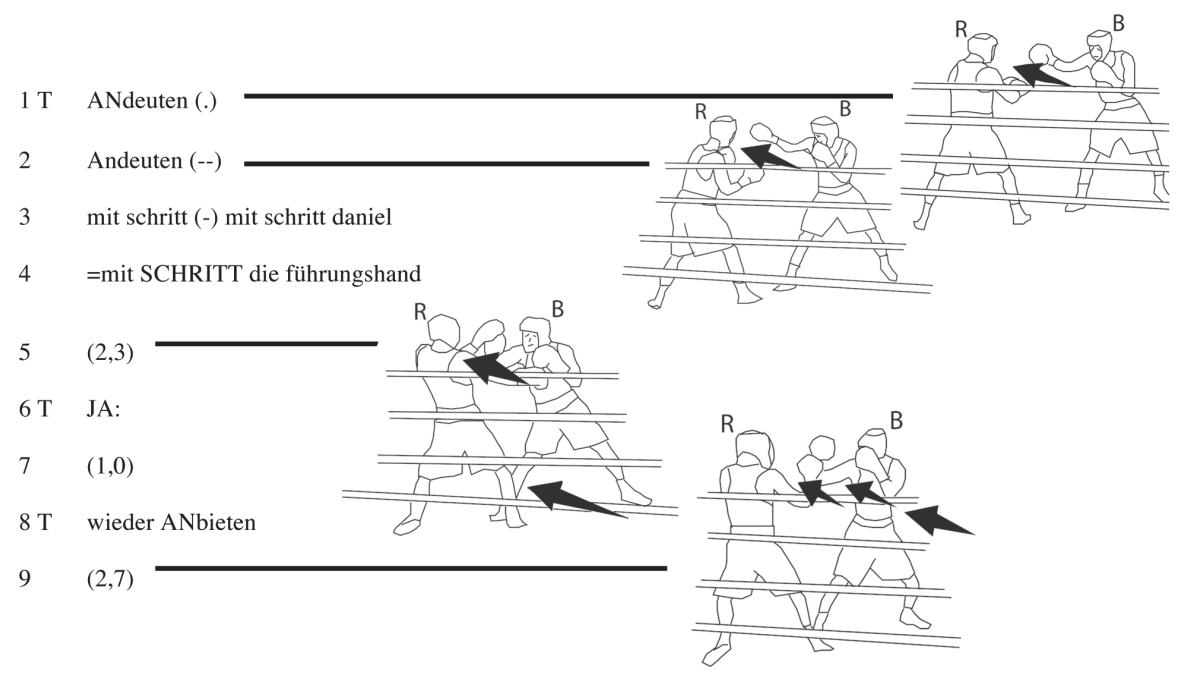

Quelle: eigene Daten 
$\mathrm{Zu}$ sehen ist hier, dass die Anweisungen des Trainers an den blauen Boxer von diesem punktgenau umgesetzt werden. In den Zeilen 1 und 2 fordert der Trainer den Boxer auf, eine Finte zu schlagen, also einen Schlag in Richtung des Gegners, der diesen aber nicht unmittelbar treffen, sondern nur zu einer Reaktion provozieren soll. Dies soll den Boxer in eine günstigere Ausgangslage für einen Angriff versetzen, etwa dass der rote Boxer seine Hände zur Deckung verwenden muss und so die Gefahr eines Gegenangriffs verringert wird. Nach seinen Anweisungen lässt der Trainer jeweils nur sehr kurze Pausen, in denen der Boxer die Aufforderung umsetzt. Dann formuliert er eine komplexere Anweisung, nämlich, dass der Boxer eine Kombination schlagen soll, die aus einer Schrittbewegung auf den Gegner zu und einem Schlag mit der Führungshand besteht (Zeilen 3 und 4). Nach dieser Aufforderung lässt er verbal eine längere Pause (Zeile 5), in der der Boxer die Anweisung umsetzt: Er macht einen Schritt auf den Gegner zu und landet mit der Führungshand einen Treffer. Dies kommentiert der Trainer mit einem langgezogenen ,JA:' (Zeile 6). Das gleiche Muster zeigt sich in Zeile 8, als der Trainer die Anweisung zum Anbieten an den Boxer gibt. In der darauffolgenden Pause (Zeile 9) setzt der Boxer dies exakt um und zeigt dadurch erneut visuell an, dass er die Anweisung des Trainers gehört und verstanden hat.

Erkennbar wird, dass der Trainer seine verbalen Beiträge sehr eng auf die sichtbaren körperlichen Aktivitäten des Boxers abstimmt, während der Boxer eben diese Aktivitäten in hohem Maße an den verbalen Beiträgen des Trainers ausrichtet. Dabei liegen hier sehr verschiedene Aktivitäten vor, die auch sehr unterschiedlich verarbeitet werden. Die Finte zu Beginn des Transkripts kann der Boxer relativ gefahrlos anbringen, da der Abstand zwischen den beiden Boxern groß genug ist, um nicht einen sofortigen Gegenschlag fürchten zu müssen. Die anschließende komplexere Anweisung kann der Boxer jedoch nicht direkt umsetzen. Hierfür braucht er mehr Zeit, da er den Gegner genau beobachten muss, um dessen mögliches Verhalten in Reaktion auf einen Angriff antizipieren zu können. Dem trägt auch der Trainer Rechnung, indem er nach der Formulierung seiner Anweisungen eine längere Pause lässt. Nach der Umsetzung der Anweisung bestätigt der Trainer die Handlung und formuliert eine neue Anweisung. Trainer und Boxer bilden hier eine Handlungseinheit, bei der die visuellen Wahrnehmungsleistungen und taktischen Planungen von zwei Personen mit den körperlichen Aktivitäten des Boxers und dessen Gegners koordiniert werden müssen. Bei der hier gezeigten Szene handelt es sich um einen Ausschnitt aus einer relativ ruhigen Kampfphase, in der der blaue Boxer überlegen ist. Doch trotz dieser Einschränkung zeigt sich darin ein prinzipieller Mechanismus des Boxens: Taktische Handlungsentscheidungen ebenso wie die visuelle Wahrnehmung, die ihnen zugrunde liegt, gehen vom Trainer aus und werden idealerweise im Anschluss physisch vom Boxer umgesetzt.

Um diese enge Verbindung zum Boxer aufrechterhalten zu können, muss der Trainer verschiedene Ebenen bedienen. Hierzu zählen Anleitungen zum generellen taktischen Vorgehen oder in Bezug auf für ihn erkennbare Muster, die Erweiterung des visuellen Felds des Boxers durch die Wahrnehmung des Trainers, aber auch die evaluative oder motivierende Ansprache. Die Koordination zwischen diesen verschiedenen Ebenen - die vor allem in einer Koordination zwischen elaboriert verbalen, paraverbal-analogen, gestischen oder anderen verkörperten Beiträgen des Trainers sichtbar wird - folgt dabei vor allem der Kontingenz des Boxgeschehens, das sich innerhalb von Sekundenbruchteilen ändert und sich damit von anderen sozialen Handlungen wie etwa dem chirurgischen Eingriff unterscheidet, die einer stärkeren Kontrolle durch einen der Beteiligten unterliegen und bei denen daher eine systematische Ausrichtung an Anweisungen geschehen kann (vgl. Mondada 2010: 220). Der schnelllebigen Kontingenz des Boxgeschehens trägt der Trainer vor allem über die extreme Verkürzung und Geschwindigkeit seiner Beiträge Rechnung, die diese erst für den Boxer umsetzbar, mit dem Boxgeschehen kommensurabel machen und so eine verteilte visuelle Praxis ermöglichen. 
Die Verbindung zwischen Boxer und Trainer ist jedoch nicht statisch, sondern sehr fragil. Um sie aufrechtzuerhalten, bedarf es einer kontinuierlichen Hervorbringungsleistung durch Boxer und Trainer. Das folgende Beispiel zeigt einen Moment, in dem die Verbindung zerfällt, was eine Reparatur nötig macht.

Transkript 13 (T: Trainer, A: Assistent)

$1 \mathrm{~T}$ oh oh oh oh ( )
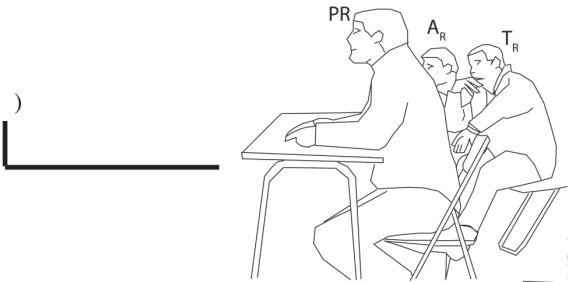

$=i c h$ weß och nich [was mit dem LOS is]

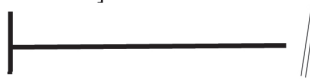

$3 \mathrm{~A}$

[weß ich auch nich( )]

$4 \mathrm{~T}$ he?

5 A (ich hab jetzt auch nich [

)]

$6 \mathrm{~T}$
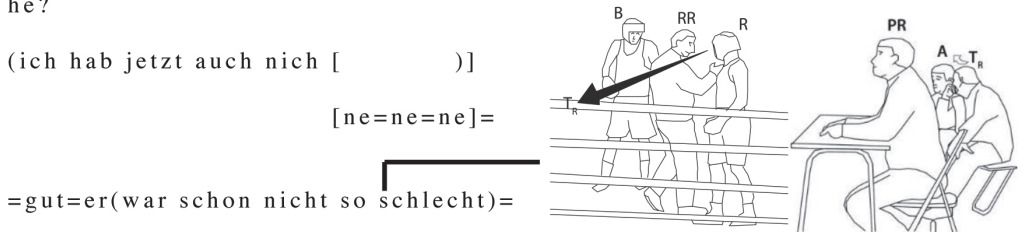

7
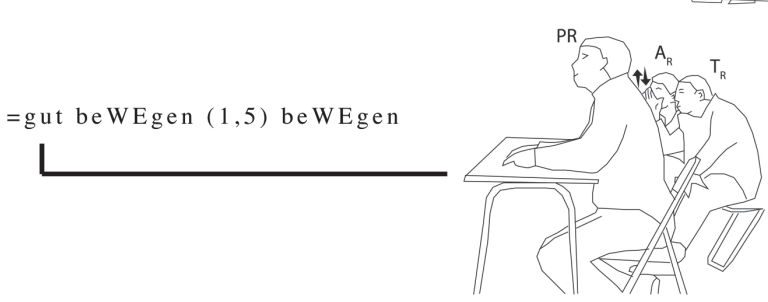

Quelle: eigene Daten

Anstatt die Aktivitäten seines Boxers zu beobachten und diesem Anweisungen in den Ring zu rufen, spricht der Trainer leise mit seinem Assistenten und wendet sich diesem auch körperlich und mit seinem Blick zu (,he?'), als er dessen Äußerung nicht verstanden hat (Zeile 1 bis 7, Standbild Zeile 7). Im Ring wurde derweil der Kampf aufgrund eines Klammerns unterbrochen. Der Boxer blickt jedoch nicht in Richtung seines Gegners, sondern zu seinem Trainer, der sich zu diesem Zeitpunkt immer noch im Gespräch befindet. Als der Trainer wieder zurück in den Ring schaut, sieht er, dass der Boxer ihn anblickt. Daraufhin löst er sich aus dem Gespräch und ruft wieder laut Anweisungen (, bewegen, bewegen!') in den Boxring. Zusätzlich hebt er seine rechte Hand und macht eine Winkgeste (Standbild Zeile 8).

Der Trainer hat seine Aufmerksamkeit auf das Kampfgeschehen unterbrochen und bespricht mit seinem Assistenten die Leistung seines Boxers. In gewisser Weise kollidiert hier das skopische Regime des Boxkampfes, das die visuelle Aufmerksamkeit des Trainers auf den Ring fordert, mit dem skopischen Regime der Interaktion, das visuelle Aufmerksamkeit insbeson- 
dere bei einer Stockung durch Nichtverstehen auf den Interaktionspartner verlangt. Erst als er die Suche nach Blickkontakt von Seiten des Boxers bemerkt, setzt er seine Anweisungen an diesen wieder fort. Dass der Boxer seine visuelle Aufmerksamkeit auf den Trainer richtet, ist extrem ungewöhnlich und auch gefährlich, da er einen Angriff seines Gegners nicht früh genug erkennen könnte. Obwohl der Kampf in dem Moment, in dem der Boxer in Richtung seines Trainers blickt, unterbrochen ist, stellt dieser Seitenblick des Boxers immer noch einen bedeutsamen Moment visueller Kommunikation (ein so genanntes „,noticeable“, vgl. Schegloff 2007: 86 f) dar. Durch den Blick des Boxers auf den Trainer verlagert sich der Aufmerksamkeitsfokus (vgl. Goodwin 2012: 413) der kooperativen Einheit auf den Trainer. Dieser Lesart entspricht auch die Reaktion des Trainers, der seine Äußerung zum Assistenten abbricht und sich verbal und mit einer Geste wieder dem Boxer zuwendet. Gestisch-visuelle Kommunikation zwischen Trainer und Boxer wird ansonsten aufgrund seiner Gefahr für den Boxer nicht ausgeübt. Es handelt sich also um einen Versuch der Reparatur der abgebrochenen Verbindung zu seinem Boxer.

\section{Fazit}

Wie gezeigt wurde, überlagern sich in der kopräsent-physischen Praxis des Boxens vielerlei skopisch-visuelle Praktiken, die sich an unterschiedliche Adressaten richten. Dass sich in der sportlichen Praxis eine Vielzahl dieser Praktiken findet, kann als Argument gegen medienkritische und kulturpessimistische Annahmen gewertet werden, dass gegenwärtig eine Tendenz zur entfremdenden und entkörperlichenden Skopisierung der sozialen Welt zu beobachten ist (vgl. Fußnote 3). Ein Unterschied besteht sicherlich darin, dass die visuellen Praktiken, die unter der interaktionalen Bedingung körperlicher Kopräsenz zum Einsatz kommen, multimodal mit anderen Modalitäten und semiotischen Ressourcen menschlicher Leibhaftigkeit wie dem Verbalen und Paraverbalen, dem Gestischen und dem Physischen verwoben sind. Angesichts der vielgestaltigen und ineinander verschachtelten visuellen Praktiken, die im Rahmen von spezifischen Sichtbarkeitserfordernissen und skopischen Regimen permanent vollzogen werden, kann mit Recht also in Bezug auf die körperliche Interaktion des Boxens und vermutlich auch darüber hinaus von einer skopischen Sozialität gesprochen werden.

Im Detail zählen sowohl die ostentative Umsetzung der permanent vokal in den Ring gerufenen Anweisung des Trainers durch den Boxer (Transkript 12) als auch seine demonstrative, an den Ringrichter adressierte Einhaltung der Boxregeln (Transkripte 4 und 5), seine betonte Performanz des Treffens beim Schlag (oder des Nichtgetroffenseins beim Schlag des Gegners) (Transkripte 2 und 3) und seine Zurschaustellung von sportlicher Fitness, moralischer Integrität und uneingeschränkter Vitalität gegenüber dem Publikum, den Punktrichtern und dem Gegner (Transkripte 6, 7 und 8) zu den visuellen, an spezifischen kontextuellen Sicht- und Unsichtbarkeitserfordernissen orientierten Praktiken der Boxer. Ein sehr basales Unsichtbarkeitserfordernis betrifft zudem das Verdecken und Antäuschen von Schlägen gegenüber dem gegnerischen Boxer. All diese Sichtbarkeitsaktivitäten müssen, jeweils adressatenspezifisch, vom Boxer in seinen Einzelhandlungen gewissermaßen , aufgeschichtet“ werden, wobei eine Vielzahl dieser Sichtbarkeiten gegenüber dem einen Adressaten nach Möglichkeit die gleichzeitige Unsichtbarkeit gegenüber dem anderen erfordert. Aufgrund des reflexiven Charakters sozialen Handelns werden im Boxen jedoch nicht nur unterschiedlich adressierte oder gar gesplittete Sichtbarkeitspraktiken ausgeübt, sondern die unterschiedlichen visuellen Praktiken werden auch zur Täuschung des Gegners ausgebeutet (Transkript 9). In der hochkomplexen und von der besonderen Temporalstruktur der extremen Schnelllebigkeit geprägten Situation des Boxens wird darüber hinaus die visuelle Konstitution der sozialen Realität bisweilen auf mehrere Personen - etwa Trainer und Boxer - verteilt, um die Sehfähigkeit beider zu steigern und den Umgang mit der physischen Realität zu verbessern (Transkript 10 und 11). 
Die genannte „Aufschichtung visueller Praxis“ umfasst sowohl kooperative als auch antagonistische Praktiken der Sichtbarmachung, wobei Erstere zu bestimmten Zeitpunkten zwischen den Interaktionspartnern koordinativ synchronisiert werden müssen, während Zweitere sich durch die Anwendung von Täuschungspraktiken auszeichnen. Beides passiert mit Rückgriff auf die multimodalen semiotischen Ressourcen, die der Leib des Boxers sowie die Materialität und Medialität der Umgebung zur Verfügung stellt. Da der boxende Leib jedoch zugleich ein biologischer Körper ist, kommt eine weitere Dimension der Sichtbarkeit hinzu: die „karnale“ Dimension, die es fast unumgänglich macht, dass auch hier Sichtbarkeiten (etwa Cuts am Auge) entstehen, die von den Beteiligten als Dokument eines Ereignisses oder Zustands (eines kassierten Schlages, körperlicher Unterlegenheit) gedeutet werden. Neben den körperlichen Handlungen wird daher auch die karnale Dimension bestimmten Sichtbarkeiten erzeugenden bzw. reduzierenden Praktiken unterworfen. Sogar für das Boxen - eine scheinbar auf die physische Konfrontation reduzierte Aktivität - gilt, dass visuelle und skopische Praktiken omnipräsent, zugleich aber reflexiv, situiert, verteilt und verkörpert sind.

Es hat sich also gezeigt, dass visuelle und skopische Praxis in der Tat nicht nur in einem physiologisch-intrapersonalen Prozess besteht, der auf pauschalen sensorischen Präferenzen beruht. Vielmehr stellt sich das Sehen in seinem Vollzug als eine kooperativ hervorgebrachte heterogene Institution dar, die sich jeweils entsprechend ihrem Kontext in einem spezifischen multimodal organisierten Zusammenspiel körperlicher und gegenständlicher Materialität aufschichtet. Obgleich er spezifische obligatorische Passagepunkte durchläuft (etwa die Redimensionalisierung durch den Ringrichter, Transkript 1), die einen definierten Anfangs- und Endpunkt haben, ist dieser kooperative Prozess der Hervorbringung von Sehen und Sichtbarkeit nicht zu einem bestimmten Zeitpunkt abgeschlossen, aus dem dann gewissermaßen eine träge fortlaufende Sozialität erwächst. Vielmehr muss er kontinuierlich weitergeführt werden, um die - stark von der Alltagswelt der meisten Menschen abweichende (Austausch körperlicher Gewalt, Wettkampfbedingungen etc.) - Situationsdefinition des Boxens aufrechtzuerhalten. Wird dieser Prozess beendet, endet auch die Situationsdefinition und die Realität wird für die Beteiligten brüchig (Transkript 13).

Das spezifische skopische Regime des Boxens betrifft somit weniger die sehr generellen Präferenzen und Tendenzen - etwa das im Lehrbuch niedergelegte und im Boxtraining eingeübte „richtige Blicken“, um Schläge des Gegners frühzeitig zu erkennen - als vielmehr vielfältige, multimodale und zum Teil multiadressierte Praktiken, die auf bestimmte Sichtbarkeitserfordernisse bezogen sind, die das Boxen bietet. Diese Sichtbarkeitserfordernisse sind vom Regelsystem gestaltet und von der Institutionalisierung des Boxsports geprägt.

Indem er ein spezifisches empirisches Beispiel intensiv beleuchtet, schlägt der vorliegende Text auch eine methodische Lösung für die Erforschung visueller sozialer Praktiken vor. Bei der Erforschung situierter verkörperter Aktivitäten (Goodwin 2000 a; Streeck / Mehus 2005; Gibson et al. 2011) erscheint es unumgänglich, erstens Wert auf die detaillierte Transkription der gesamten multimodalen Äußerungen und Praktiken, darunter im besonderen Maße auch der visuellen Aktivitäten zu legen - was freilich bislang noch nicht in all seinen Facetten erprobt und in einem endgültigen Standard niedergelegt wurde und daher vorläufig zu einem gewissen Grad noch experimentell bleiben muss. Zweitens wird über die registrierende Konservierung des sozialen Geschehens mittels multiperspektivischer audiovisueller Aufzeichnungen die Temporalstruktur der sequenziell organisierten Vollzugswirklichkeit bewahrt (Bergmann 1985). Dementsprechend kann in der Analyse auch das Geschehene in seiner inkrementellen Sequenzialität rekonstruiert und in seiner Handlungslogik nachvollzogen werden. 


\section{Literatur}

Bergmann, J.R. (1985): Flüchtigkeit und methodische Fixierung sozialer Wirklichkeit Aufzeichnungen als Daten der interpretativen Soziologie, in: W. Bonß / H. Hartmann (Hrsg.), Entzauberte Wissenschaft. Zur Relativität und Geltung soziologischer Forschung (Sonderband 3 der Zeitschrift Soziale Welt 36). Göttingen, S. 299-320.

Foucault, M. (1976): Überwachen und Strafen: Die Geburt des Gefängnisses, Frankfurt / Main.

Gibson, W. / H. Webb / D. vom Lehn (2011): Re-Constituting Social Praxis: An Ethnomethodological Analysis of Video Data in Optometry Consultations, in: International Journal of Social Research Methodology 14, S. 207-218.

Goodwin, C. (1980): Restarts, Pauses, and the Achievement of Mutual Gaze at Turn-Beginning, in: Sociological Inquiry 50, S. 272-302.

Goodwin, C: (1994): Professional Vision, in: American Anthropologist 96, S. 606-633.

Goodwin, C. (1995): Seeing in Depth, in: Social Studies of Science 25, S. 237-274.

Goodwin, C. (1996): Transparent Vision, in: E. Ochs / E.A. Schegloff / S. Thompson (Hrsg.), Interaction and Grammar, Cambridge, S. 370-404.

Goodwin, C. (2000 a): Action and embodiment within situated human interaction, in: Journal of Pragmatics 32, S. $1489-1522$.

Goodwin, C. (2000 b): Practices of Seeing, Visual Analysis: An Ethnomethodological Approach, in: T. van Leeuwen / C. Jewitt (Hrsg.), Handbook of Visual Analysis, London, S. 157-182.

Goodwin, C. (2000 c): Vision, in: Journal of Linguistic Anthropology 9, S. 267-270.

Goodwin, C. (2003): Pointing as Situated Practice, in: S. Kita (Hrsg.), Pointing: Where Language, Culture and Cognition Meet, Mahwah / NJ, S. 217-41.

Goodwin, C. (2012): Zeigegesten und kollaborative Bedeutungskonstitution in der Interaktion mit Aphasikern, in: R. Ayaß / C. Meyer (Hrsg.), Sozialität in Slow Motion, Wiesbaden, S. 405-418.

Goodwin, C. / M. Goodwin (1996): Formulating Planes: Seeing as a Situated Activity, in: Y. Engestrom / D. Middleton (Hrsg.), Cognition and Communication at Work, Cambridge, S. 61-95.

Goodwin, C. / M. Goodwin (1997): Contested Vision: The Discursive Constitution of Rodney King, in: B.-L. Gunnarsson / P. Linell / B. Nordberg (Hrsg.), The Construction of Professional Discourse, New York / NY, S. 292-316.

Hausendorf, H. / R. Schmitt (2010): Opening up Openings: Zur Struktur der Eröffnungsphase eines Gottesdienstes, in: L. Mondada / R. Schmitt (Hrsg.), Situationseröffnungen: Zur multimodalen Herstellung fokussierter Interaktion, Tübingen, S. 53-102.

Hester, S. / D. Francis (2003): Analysing Visually Available Mundane Order: A Walk to the Supermarket, in: M. Ball (Hrsg.), Image Work (Sonderheft Visual Studies 18 / 1), S. 36-46.

Hutchins, E. (1995): Cognition in the Wild, Cambridge / MA.

Jay, M. (1988): Scopic Regimes of Modernity, in: H. Foster (Hrsg.), Vision and Visuality, Seattle, S. 3-28.

Kita, S. (Hrsg.) (2003): Pointing: Where Language, Culture, and Cognition Meet, Mahwah / NJ.

Knorr Cetina, K. (2012): Die synthetische Situation, in: R. Ayaß / C. Meyer (Hrsg.), Sozialität in Slow Motion, Wiesbaden, S. 81-109.

Levin, D. M. (1988): The Opening of Vision: Nihilism and the Postmodern Situation, New York / NY.

Macbeth, D. (1999): Glances, Trances, and their Relevance for a Visual Sociology, in: P.L. Jalbert (Hrsg.), Media Studies: Ethnomethodological Approaches, Lanham / NY, S. 135-170.

Metz, C. (1982): Psychoanalysis and Cinema. The Imaginary Signifier, London.

Mitchell, W.J.T. (2002): Showing Seeing: A Critique of Visual Culture, in: Journal of Visual Culture 1, S. 165-181. 
Mondada, L. (2011): The Organization of Concurrent Courses of Action in Surgical Demonstrations, in: J. Streeck / C. Goodwin / C. LeBaron (Hrsg.), Embodied Interaction - Language and Body in the Material World, Cambridge, S. 207-226.

Rorty, R. (1981): Der Spiegel der Natur: eine Kritik der Philosophie, Frankfurt / Main.

Sacks, H. / E.A. Schegloff / G. Jefferson (1974): A Simplest Systematics for the Organization of Turntaking in Conversation, in: Language 50, S. 696-735.

Schegloff, E.A. (2007): A Primer in Conversation Analysis / 1: Sequence Organization in Interaction, Cambridge.

Schütz, A. / T. Luckmann (2003): Strukturen der Lebenswelt, Konstanz.

Smith, M. (2008): Introduction. Visual Culture Studies: History, Theory, Practice, in: Ders. (Hrsg.), Visual Culture Studies, London, S. 1-16.

Spitzer, M. (2006): Vorsicht Bildschirm! Elektronische Medien, Gehirnentwicklung, Gesundheit und Gesellschaft, München.

Streeck, J. / S. Mehus (2005): Microethnography: The Study of Practices, in: K.L. Fitch / R.E. Sanders (Hrsg.), Handbook of Language and Social Interaction, Mahwah / NJ, S. 381-404.

Tyler, S.A. (1984): Vision Quest in the West. What the Mind's Eye Sees, in: Journal of Anthropological Research 40, S. 23-40.

Wolff, S. (1994): Text und Schuld: die Rhetorik psychiatrischer Gerichtsgutachten, Berlin.

PD Dr. Christian Meyer Universität Duisburg-Essen Global Cooperation Research Center Schifferstraße 196 47059 Duisburg christian.meyer5@uni-bielefeld.de

Ulrich von Wedelstaedt Universität Bielefeld

Fakultät für Soziologie Postfach 100131 33501 Bielefeld ulrich.wedelstaedt@uni-bielefeld.de 


\section{Anhang: Verwendete Transkriptionszeichen, Lemmata und Siglen}

\begin{tabular}{|c|c|c|}
\hline $\mathrm{R}$ & \multicolumn{2}{|l|}{ Roter Boxer } \\
\hline B & \multicolumn{2}{|c|}{ Blauer Boxer } \\
\hline RR & \multicolumn{2}{|c|}{ Ringrichter (in den Standbildern jeweils ohne Bezeichnung) } \\
\hline $\mathrm{T}_{\mathrm{R}}$ & \multicolumn{2}{|c|}{ Trainer des roten Boxers } \\
\hline $\mathrm{T}_{\mathrm{B}}$ & \multicolumn{2}{|c|}{ Trainer des blauen Boxers } \\
\hline$A_{R}$ & \multicolumn{2}{|c|}{ Assistent des roten Trainers } \\
\hline$A_{B}$ & \multicolumn{2}{|c|}{ Assistent des blauen Trainers } \\
\hline PR & \multicolumn{2}{|c|}{ Punktrichter } \\
\hline (.) & \multicolumn{2}{|l|}{ Mikropause } \\
\hline$(-) ;(--) ;(--)$ & \multicolumn{2}{|c|}{ Pausen von ca. 0,$25 ; 0,5 ; 0,75$ Sek. Länge (geschätzt) } \\
\hline$(1,5)$ & \multicolumn{2}{|c|}{ Pause in gemessener Länge } \\
\hline$=$ & \multicolumn{2}{|c|}{ unmittelbarer Anschluss } \\
\hline & \multicolumn{2}{|c|}{ Vokallängung } \\
\hline beTONUNG & \multicolumn{2}{|c|}{ Betonte Silben in Großschrift } \\
\hline .h & \multicolumn{2}{|c|}{ hörbares kurzes Einatmen } \\
\hline$(($ klatscht $))$ & \multicolumn{2}{|c|}{ Paraverbale Äußerungen und Ereignisse } \\
\hline ( ) & \multicolumn{2}{|c|}{ Unverständliche Passage } \\
\hline (selber) & \multicolumn{2}{|l|}{ Unsichere Transkription } \\
\hline [überlappung] & \multicolumn{2}{|c|}{ Überlappung mit der entsprechenden Passage in der nächsten Zeile } \\
\hline Pfeil am Gesicl & & Blickrichtung \\
\hline Pfeil an Beinen & apf oder über dem Kopf & Bewegungsrichtung eines Körpers \\
\hline Pfeil an den Hä & & Bewegungsrichtung der Hände \\
\hline Doppelte Pfeile & & Bewegungen schnellerer Geschwin \\
\hline
\end{tabular}

